\title{
RELAXATION AND NUMERICAL APPROXIMATION OF A TWO-FLUID TWO-PRESSURE DIPHASIC MODEL
}

\author{
Annalisa Ambroso ${ }^{1}$, Christophe Chalons $^{1,2}$, Frédéric Coquel ${ }^{3,4}$ \\ And Thomas Galié ${ }^{1}$
}

\begin{abstract}
This paper is concerned with the numerical approximation of the solutions of a two-fluid two-pressure model used in the modelling of two-phase flows. We present a relaxation strategy for easily dealing with both the nonlinearities associated with the pressure laws and the nonconservative terms that are inherently present in the set of convective equations and that couple the two phases. In particular, the proposed approximate Riemann solver is given by explicit formulas, preserves the natural phase space, and exactly captures the coupling waves between the two phases. Numerical evidences are given to corroborate the validity of our approach.
\end{abstract}

Mathematics Subject Classification. 76T10, 35L60, 76M12.

Received May 6, 2008. Revised November 3rd, 2008.

Published online October 9, 2009.

\section{INTRODUCTION}

In this paper, we are interested in the numerical approximation of the solutions of a two fluid two pressure diphasic model. That kind of model has gained interest in the recent years for the modelling and computation of two phase flows. It was first formulated in Baer and Nunziato [6] and its mathematical properties have been first addressed in Embid and Baer [18]. This model was subsequently studied in Kapila et al. [27], Glimm et al. [22], Saurel and Abgrall [35], Gavrilyuk and Saurel [21], Gallouët et al. [20], Coquel et al. [16]. It treats each phase as a separate fluid meaning that each phase $k$ has its own density $\rho_{k}$, velocity $u_{k}$ and pressure $p_{k}$ with $k=1,2$. In particular, the velocity and pressure nonequilibria between the phases are not neglected.

In the case of a barotropic flow (the pressure is for each phase a function of the density only), the basic set of convective equations is composed of the classical partial differential equations governing the mass $\alpha_{k} \rho_{k}$ and the momentum $\alpha_{k} \rho_{k} u_{k}$. Here $\alpha_{k}$ is the volume fraction of phase $k$ and the two fluids are non-miscible, which implies $\alpha_{1}+\alpha_{2}=1$. The coupling between the two phases is made by two nonconservative terms

\footnotetext{
Keywords and phrases. Two-phase flows, two-fluid two-pressure model, hyperbolic systems, finite volume methods, relaxation schemes, Riemann solvers.

1 DEN/DANS/DM2S/SFME/LETR CEA-Saclay, 91191 Gif-sur-Yvette, France. annalisa.ambroso@cea.fr

2 Université Paris 7-Denis Diderot and UMR 7598, Laboratoire Jacques-Louis Lions, 75005 Paris, France.

chalons@math.jussieu.fr

3 Université Pierre et Marie Curie-Paris 6, UMR 7598, Laboratoire Jacques-Louis Lions, 75005 Paris, France.

coquel@ann. jussieu.fr

4 CNRS, UMR 7598, Laboratoire Jacques-Louis Lions, 75005 Paris, France.
} 
$p_{I} \partial_{x} \alpha_{k}, k=1,2$ involving the interfacial pressure $p_{I}$ in the momentum equations, whereas $\alpha_{k}$ evolves according to its own equation. More precisely, it is advected with the interfacial velocity $u_{I}$. Several choices for $u_{I}$ and $p_{I}$ can be found in the literature. Here we choose to set

$$
u_{I}=u_{2}, \quad p_{I}=p_{1}\left(\rho_{1}\right)
$$

following $[6,16,21]$. This closure was first proposed for modelling a fluid-solid interface in a detonation model, but it applies also to our case of a liquid-vapor interface provided that one of the phases is dispersed, i.e. its concentration is small. Other closure laws has been proposed for fluid-fluid models, see for instance [35]. We refer the reader to Gallouët et al. [20] for a comprehensive study of general closure laws concerning $u_{I}$ and $p_{I}$. Relations (0.1) ensure that the void fraction wave is linearly degenerate. Source terms like gravity force and pressure relaxation will be added in the equations in the numerical part of the paper to simulate typical two phase problems. The problem of the relaxation of the fluid velocities is not addressed here. We study that problem in [2].

Importantly, the model under consideration admits systematically five real eigenvalues and is seen to have a basis of right eigenvectors, at least in the context of subsonic flows. From a mathematical viewpoint, this flow regime expresses that some of the eigenvalues do not coincide and is completely relevant in the nuclear energy industry framework which motivates this work. The hyperbolicity property makes the two-fluid two-pressure approach very attractive in comparison to models using an equilibrium pressure assumption $\left(p=p_{1}=p_{2}\right)$ since the latter do not necessarily admit real eigenvalues in all situations.

The resolution of the proposed system is certainly not easy, for two main reasons. The first one comes from the (possibly) strong nonlinearities associated with the pressure laws $p_{k}, k=1,2$. In order to overcome this difficulty, we propose in this work a numerical scheme which is based on a relaxation approach. The idea is to approximate at the discrete level the solutions of the system (the so-called equilibrium system) by those of a suitable extended first order system with singular perturbation (the so-called relaxation system). See for instance Jin and Xin [26], Chalons and Coquel [12], Coquel et al. [15], Chalons and Coulombel [13]. This relaxation system has the property of being nonlinear hyperbolic with only linearly degenerate characteristic fields, which makes the numerical resolution of the equilibrium system easier.

The second difficulty stems from the presence of the nonconservative terms $p_{I} \partial_{x} \alpha_{k}, k=1,2$ which implies that the (equilibrium) system does not admit any equivalent conservation form in general. Of course, these nonconservative terms vanish in the very particular situation in which $\alpha_{k}$ is locally constant and the structure becomes the one of two conservative and decoupled two by two Euler systems. In the general case, the choice (0.1), by ensuring that the void fraction wave is linearly degenerate, allows to give sense to the nonconservative terms. By contrast with nonconservative products appearing in shocks $[29,30]$, nonconservative products propagated by contact discontinuities do not depend on the underlying viscous phenomena. With this respect, discrete solutions do not depend on numerical viscosity (Guillemaud [25]). Difficulties may arise but they are linked with the appearance of resonance phenomena (Andrianov and Warnecke [4], Andrianov [3]...). In this context, we derive in this work an approximate Riemann solver so as to preserve in the best possible way the contact discontinuity $u_{I}$ associated with the initial system, and this is achieved by a particular treatment of the nonconservative products in the relaxation system. We prove that the proposed Riemann solver is conservative for the mass of each phase and for the total momentum, and that it captures exactly the $u_{I}$-contact discontinuities associated with the original system. In addition, it is given by fully explicit formulas and is stable in the $L^{1}$ sense (i.e. it preserves the natural phase space).

One may find in the literature several quite recent papers devoted to the numerical resolution of two-fluid two-pressure models and the question of how to discretize the nonconservative terms. Most of them deal with the non barotropic case. Actually, the literature is large on this subject and we do not claim that the following discussion is exhaustive. We refer in particular to the numerous works quoted in the references we mention. A first group of works is due to Saurel and collaborators. Saurel and Abgrall in [35] and Andrianov et al. in [5] for instance (see also Saurel and Lemetayer [36] for a multidimensional framework) take into account the nonconservative terms by means of a free streaming physical condition associated with uniform velocity 
and pressure profiles. The discretization technique of [35] is improved by the same authors in [1] in an original way. It consists in averaging some approximations of the pure phase Euler equations at the microscopic level instead of, as it is more usual, approximating the averaged multiphase flow equations. Their goal is to account for the volume fraction variations inside the shock relations in presence of source terms. In Andrianov and Warnecke [4] and Schwendeman et al. [37], the common objective is to get exact solutions for the Riemann problem of the model. The approach is inverse in [4] in the sense that the initial left and right states are obtained as function of the intermediate states of the solution. On the contrary, a direct iterative approach is used in [37] leading to exact solutions of the Riemann problem for any initial left and right states. Another direct approach to construct theoretical solutions is proposed in Castro and Toro [11]. In this work the authors propose to solve the Riemann problem approximately assuming that all the nonlinear characteristic fields are associated with rarefaction waves. Finally, all these (approximate or exact) solutions are used to develop a Godunov-type method. At last, other finite volumes methods have been used. For instance in Gallouët et al. [20] (see also Guillemaud [25]), the approximation of the convective terms of the system is based on the Rusanov scheme (Rusanov [34]) and the so-called VFRoe-ncv scheme (Buffard et al. [10]), these strategies being adapted to the nonconservative framework. In Munkejord [32] and Karni et al. [28], the author use Roe-type schemes.

The outline of the paper is as follows. Section 1 introduces some notations, gives the governing equations of the two-fluid two-pressure model and states its basic properties. Section 2 characterizes the admissible contact discontinuities associated with the coupling wave $u_{I}$. The next two sections are devoted to the relaxation approximation of the two-fluid two-pressure model and follow the same lines. Section 3 thus describes the relaxation system and gives its basic properties whereas Section 4 proposes a characterization of the corresponding admissible $u_{I}$-contact discontinuities. At last, Section 5 describes the approximate Riemann solver and the relaxation scheme, and Section 6 is devoted to numerical experiments.

To conclude this introduction, let us mention that this work falls within the scope of a joint research program on the coupling of multiphase flow models between CEA-Saclay and Laboratoire Jacques-Louis Lions ${ }^{5}$. In the short term, the objective of the working group is to couple a two-fluid two-pressure model and a drift-flux model.

\section{Governing EQUATions}

In one space dimension, the convective part of the model under consideration in this paper reads:

$$
\left\{\begin{array}{l}
\partial_{t} \alpha_{1}+u_{I} \partial_{x} \alpha_{1}=0, \\
\partial_{t} \alpha_{1} \rho_{1}+\partial_{x}\left(\alpha_{1} \rho_{1} u_{1}\right)=0, \\
\partial_{t} \alpha_{1} \rho_{1} u_{1}+\partial_{x}\left(\alpha_{1} \rho_{1} u_{1}^{2}+\alpha_{1} p_{1}\left(\rho_{1}\right)\right)-p_{I} \partial_{x} \alpha_{1}=0, \quad t>0, \quad x \in \mathbb{R}, \\
\partial_{t} \alpha_{2} \rho_{2}+\partial_{x}\left(\alpha_{2} \rho_{2} u_{2}\right)=0, \\
\partial_{t} \alpha_{2} \rho_{2} u_{2}+\partial_{x}\left(\alpha_{2} \rho_{2} u_{2}^{2}+\alpha_{2} p_{2}\left(\rho_{2}\right)\right)+p_{I} \partial_{x} \alpha_{1}=0 .
\end{array}\right.
$$

It takes the following condensed form:

$$
\partial_{t} \mathbf{u}+\partial_{x} \mathbf{f}(\mathbf{u})+\mathbf{c}(\mathbf{u}) \partial_{x} \mathbf{u}=0, \quad t>0, \quad x \in \mathbb{R}
$$

where $t$ is the time and $x$ is the space variable. The unknown vector $\mathbf{u}$ is defined by

$$
\mathbf{u}=\left(\begin{array}{c}
\alpha_{1} \\
\mathbf{u}_{1} \\
\mathbf{u}_{2}
\end{array}\right), \quad \text { with } \quad \mathbf{u}_{1}=\left(\begin{array}{c}
\alpha_{1} \rho_{1} \\
\alpha_{1} \rho_{1} u_{1}
\end{array}\right), \quad \mathbf{u}_{2}=\left(\begin{array}{c}
\alpha_{2} \rho_{2} \\
\alpha_{2} \rho_{2} u_{2}
\end{array}\right)
$$

and $\alpha_{1}, \rho_{1}$ and $u_{1}$ (respectively $\alpha_{2}=1-\alpha_{1}, \rho_{2}$ and $u_{2}$ ) represent the volume fraction, the density and the velocity of the phase 1 (resp. of the phase 2). This vector is expected to belong to the following natural

\footnotetext{
${ }^{5}$ See http://www.ann.jussieu.fr/groupes/cea/.
} 
phase space

$$
\Omega=\left\{\left(\alpha_{1}, \alpha_{1} \rho_{1}, \alpha_{1} \rho_{1} u_{1}, \alpha_{2} \rho_{2}, \alpha_{2} \rho_{2} u_{2}\right)^{t} \in \mathbb{R}^{5} \quad \text { such that } \quad 0<\alpha_{k}<1 \quad \text { and } \quad \rho_{k}>0 \quad \text { for } \quad k=1,2\right\} \text {. }
$$

The functions $\mathbf{f}: \Omega \rightarrow \mathbb{R}^{5}$ and $\mathbf{c}: \Omega \rightarrow \mathbb{R}^{5 \times 5}$ are such that

$$
\mathbf{f}(\mathbf{u})=\left(\begin{array}{c}
0 \\
\alpha_{1} \rho_{1} u_{1} \\
\alpha_{1} \rho_{1} u_{1}^{2}+\alpha_{1} p_{1}\left(\rho_{1}\right) \\
\alpha_{2} \rho_{2} u_{2} \\
\alpha_{2} \rho_{2} u_{2}^{2}+\alpha_{2} p_{2}\left(\rho_{2}\right)
\end{array}\right), \quad \mathbf{c}(\mathbf{u}) \partial_{x} \mathbf{u}=\left(\begin{array}{c}
u_{I} \partial_{x} \alpha_{1} \\
0 \\
-p_{I} \partial_{x} \alpha_{1} \\
0 \\
p_{I} \partial_{x} \alpha_{1}
\end{array}\right)
$$

where the pressure laws $p_{k}, k=1,2$ are given smooth functions such that $p_{k}\left(\rho_{k}\right)>0, p_{k}^{\prime}\left(\rho_{k}\right)>0, p_{k}^{\prime \prime}\left(\rho_{k}\right)+$ $\frac{2}{\rho_{k}} p_{k}^{\prime}\left(\rho_{k}\right)>0, \lim _{\rho_{k} \rightarrow 0} p_{k}\left(\rho_{k}\right)=0$ and $\lim _{\rho_{k} \rightarrow \infty} p_{k}\left(\rho_{k}\right)=+\infty$. The proposed interfacial velocity $u_{I}$ and pressure $p_{I}$ are

$$
u_{I}=u_{2}, \quad p_{I}=p_{1}\left(\rho_{1}\right),
$$

which corresponds to a particular choice of the closures proposed for instance in [20,25].

Since the fluid is adiabatic, the internal energies $e_{k}$, are defined by

$$
\mathrm{d} e_{k}\left(\rho_{k}, s_{k}\right)=-\frac{p_{k}\left(\rho_{k}\right)}{\rho_{k}^{2}} \mathrm{~d} \rho_{k}+T \mathrm{~d} s_{k}=-\frac{p_{k}\left(\rho_{k}\right)}{\rho_{k}^{2}} \mathrm{~d} \rho_{k},
$$

which gives

$$
e_{k}^{\prime}\left(\rho_{k}\right)=\frac{p_{k}\left(\rho_{k}\right)}{\rho_{k}^{2}}
$$

The total energies $E_{k}$ and the enthalpies $h_{k}$ of each phase are given by the following relations

$$
E_{k}\left(\mathbf{u}_{k}\right)=\frac{1}{2} u_{k}^{2}+e_{k}\left(\rho_{k}\right), \quad h_{k}\left(\rho_{k}\right)=e_{k}\left(\rho_{k}\right)+\frac{p_{k}\left(\rho_{k}\right)}{\rho_{k}}, \quad k=1,2
$$

The sound speeds are given by

$$
c_{k}=\sqrt{p_{k}^{\prime}\left(\rho_{k}\right)}, \quad k=1,2
$$

The following proposition holds.

Proposition 1.1. For all vector $\mathbf{u}$ in the phase space $\Omega$, system (1.2) admits the following five real eigenvalues

$$
\begin{gathered}
\lambda_{0}(\mathbf{u})=u_{2}, \\
\lambda_{1}(\mathbf{u})=u_{1}-c_{1}, \quad \lambda_{2}(\mathbf{u})=u_{1}+c_{1}, \\
\lambda_{3}(\mathbf{u})=u_{2}-c_{2}, \quad \lambda_{4}(\mathbf{u})=u_{2}+c_{2},
\end{gathered}
$$

and is hyperbolic on $\Omega$ (the corresponding right eigenvectors span $\mathbb{R}^{5}$ ) as soon as $u_{2} \neq u_{1} \pm c_{1}$. Moreover, the characteristic fields associated with $\left\{\lambda_{i}\right\}_{i=1, \ldots, 4}$ are genuinely nonlinear, whereas the characteristic field associated with $\lambda_{0}$ is linearly degenerate.

Proof. This result has already been stated in [32] in the barotropic case (in the case of the full system, see Baer and Nunziato [6]). For the sake of completeness, we briefly recall the main ingredients of its proof.

First, classical manipulations on system (1.2) show that it can be recast in the following quasi-linear form

$$
\partial_{t} \mathbf{u}+\mathbf{A}(\mathbf{u}) \partial_{x} \mathbf{u}=0
$$


where the coefficient matrix $\mathbf{A}(\mathbf{u})$ is given by

$$
\mathbf{A}(\mathbf{u})=\left(\begin{array}{ccccc}
u_{2} & 0 & 0 & 0 & 0 \\
0 & 0 & 1 & 0 & 0 \\
\chi_{1} & c_{1}^{2}-u_{1}^{2} & 2 u_{1} & 0 & 0 \\
0 & 0 & 0 & 0 & 1 \\
-\chi_{2} & 0 & 0 & c_{2}^{2}-u_{2}^{2} & 2 u_{2}
\end{array}\right)
$$

with

$$
\chi_{k}=\left(p_{k}-p_{I}\right)-\rho_{k} c_{k}^{2}, \quad k=1,2 .
$$

Recall that $u_{I}=u_{2}$ in this paper. The eigenvalues (1.7) are easily obtained using a block decomposition of matrix (1.9).

Then, provided that $u_{2} \neq u_{1} \pm c_{1}$ (that is $\lambda_{0} \neq\left\{\lambda_{i}\right\}_{i=1, \ldots, 4}$ ), the right eigenvector matrix $\mathbf{R}$ is found to be invertible and given by

$$
\mathbf{R}(\mathbf{u})=\left(\begin{array}{ccccc}
1 & 0 & 0 & 0 & 0 \\
\frac{\chi_{1}}{\left(\lambda_{1}-\lambda_{0}\right)\left(\lambda_{2}-\lambda_{0}\right)} & 1 & 1 & 0 & 0 \\
\frac{\chi_{1} u_{2}}{\left(\lambda_{1}-\lambda_{0}\right)\left(\lambda_{2}-\lambda_{0}\right)} & \lambda_{1} & \lambda_{2} & 0 & 0 \\
-\frac{\chi_{2}}{\left(\lambda_{3}-\lambda_{0}\right)\left(\lambda_{4}-\lambda_{0}\right)} & 0 & 0 & 1 & 1 \\
-\frac{\chi_{2} u_{2}}{\left(\lambda_{3}-\lambda_{0}\right)\left(\lambda_{4}-\lambda_{0}\right)} & 0 & 0 & \lambda_{3} & \lambda_{4}
\end{array}\right)
$$

At last, easy calculations give the sort of the characteristic fields associated with $\left\{\lambda_{i}\right\}_{i=1, \ldots, 4}$ (genuinely nonlinear) and $\lambda_{0}$ (linearly degenerate).

We note that system (1.2) is not always hyperbolic. When $u_{2}=u_{1} \pm c_{1}$, the coefficient matrix $\mathbf{A}(\mathbf{u})$ is no longer diagonalizable and the system becomes resonant. This is known to generate important difficulties [23,31] but such a situation will not be addressed in the present work. In fact, we are mainly interested in subsonic flows so that the restriction $u_{2} \neq u_{1} \pm c_{1}$ will be always satisfied in practice.

Note also that the characteristic field associated with the wave $\lambda_{0}$ between the two phases is linearly degenerate. This is a consequence of the particular choice (1.4) (see again $[20,25]$ for more details).

\section{Jump RELATIONS ACROSS A CONTACT DISCONTINUITY}

Solving the Riemann problem associated with (1.2) for the following initial data

$$
\mathbf{u}_{0}(x)=\left\{\begin{array}{lll}
\mathbf{u}_{-} & \text {if } & x<0 \\
\mathbf{u}_{+} & \text {if } & x>0
\end{array}\right.
$$

turns out to be a difficult question. The main point in this study is the large number of characteristic speeds (five, see the previous proposition) and the possible occurrence of resonance. However, the general structure of a Riemann solution to (1.2)-(2.1) is made of shock and rarefaction waves associated with eigenvalues $\left\{\lambda_{i}\right\}_{i=1, \ldots, 4}$ and a contact discontinuity associated with $\lambda_{0}$, see Figure 1 in the case of subsonic flows $\left(u_{k}-c_{k} \ll u_{2} \ll u_{k}+c_{k}\right.$, $k=1,2)$. From the first equation in system (1.2), the void fractions $\alpha_{1}$ and $\alpha_{2}$ are constant on the left and on the right of the latter $\lambda_{0}$-discontinuity and in these regions, system (1.2) consists of two independent Euler systems for the two phases 1 and 2 . That is why the $\lambda_{0}$-discontinuity is often called the coupling wave between phases 1 and 2. Note that due to the nonlinearities of the pressure laws $p_{k}=p_{k}\left(\rho_{k}\right), k=1,2$, the intermediate states which separate the five simple waves in such a Riemann solution are difficult to determine, at least 


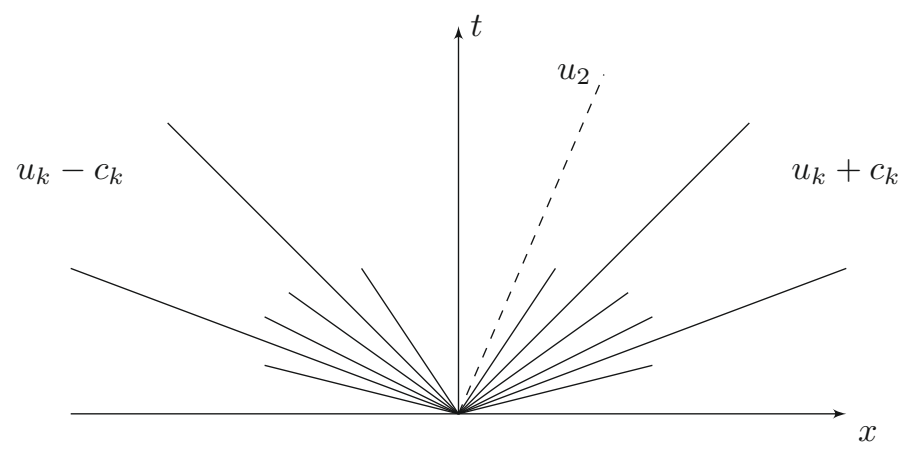

FiguRE 1. General structure in the subsonic regime of the Riemann solution associated with (1.2).

in the general situation $\alpha_{1-} \neq \alpha_{1+}$ (if $\alpha_{1-}=\alpha_{1+}$, the intermediate states follow from classical considerations on the two independent Euler systems for the two phases, see for instance [24]).

Therefore, instead of trying to solve the full Riemann problem (1.2)-(2.1), in this section we focus our attention on particular solutions made of only two constant states $\left(\mathbf{u}_{-}\right.$and $\left.\mathbf{u}_{+}\right)$separated by a contact discontinuity propagating with characteristic speed $\lambda_{0}=u_{2-}=u_{2+}$, namely the contact or coupling wave. Recall that this characteristic speed is related to a linearly degenerate field with the consequence that the non conservative product $u_{2} \partial_{x} \alpha_{1}$ is well defined in (1.3) ( $u_{2}$ remains continuous even if $\alpha_{1}$ presents a discontinuity, see also [20]). Then, in order to characterize such (admissible) solutions, four independent jump relations, or equivalently four independent $\lambda_{0}$-Riemann invariants, have to be found. We first remark that system (1.2) is clearly made of two conservation laws, the other partial differential equations being non conservative. At this stage, no more than two Rankine-Hugoniot jump relations can thus be obtained. The next statement provides two additional conservation laws satisfied by the smooth solutions of (1.2), eventually leading to a full set of jump relations.

Proposition 2.1. Smooth solutions of (1.2) obey the following two conservation laws

$$
\left\{\begin{array}{l}
\partial_{t}\left(\alpha_{1} \rho_{1} u_{1}+\alpha_{2} \rho_{2} u_{2}\right)+\partial_{x}\left(\alpha_{1} \rho_{1} u_{1}^{2}+\alpha_{2} \rho_{2} u_{2}^{2}+\alpha_{1} p_{1}\left(\rho_{1}\right)+\alpha_{2} p_{2}\left(\rho_{2}\right)\right)=0 \\
\partial_{t} u_{1}+\partial_{x}\left(\frac{1}{2} u_{1}^{2}+h_{1}\left(\rho_{1}\right)\right)=0
\end{array}\right.
$$

Proof. The proposed equations follow from classical manipulations on system (1.2) and definitions (1.5), (1.6). These are left to the reader.

The above relations hold true in the weak sense for solutions presenting contact discontinuities (see for instance [24]). We thus use the additional Rankine-Hugoniot jump relations provided by these two conservation laws to establish the following theorem.

Definition 2.2. Let $\mathbf{u}_{-}$and $\mathbf{u}_{+}$be two constant states in $\Omega$. These states can be joined by an admissible $\lambda_{0}$-contact discontinuity if and only if the following jump relations hold:

$$
\left\{\begin{array}{c}
u_{2}:=u_{2-}=u_{2+} \\
m:=\alpha_{1-} \rho_{1-}\left(u_{1-}-u_{2}\right)=\alpha_{1+} \rho_{1+}\left(u_{1+}-u_{2}\right) \\
m u_{1-}+\alpha_{1-} p_{1}\left(\rho_{1-}\right)+\alpha_{2-} p_{2}\left(\rho_{2-}\right)=m u_{1+}+\alpha_{1+} p_{1}\left(\rho_{1+}\right)+\alpha_{2+} p_{2}\left(\rho_{2+}\right) \\
\frac{m^{2}}{2 \alpha_{1-}^{2} \rho_{1-}^{2}}+h_{1}\left(\rho_{1-}\right)=\frac{m^{2}}{2 \alpha_{1+}^{2} \rho_{1+}^{2}}+h_{1}\left(\rho_{1+}\right) .
\end{array}\right.
$$

In such a situation, the speed of propagation of the discontinuity is given by $u_{2}$. 
Now it is a natural question to wonder whether being given a state $\mathbf{u}_{-} \in \Omega$ there exists a state $\mathbf{u} \in \Omega$ such that (2.2) holds with $\mathbf{u}_{+}=\mathbf{u}$. Actually, for the forthcoming developments, we prefer to answer to this question in a slightly different way, namely we show that being given $\mathbf{u}_{-} \in \Omega$ and $\alpha_{1+} \in(0,1)$, it is possible to find a state $\mathbf{u}=\left(\alpha_{1}, \mathbf{u}_{1}, \mathbf{u}_{2}\right)^{t} \in \Omega$ such that $\alpha_{1}=\alpha_{1+}$ and $(2.2)$ holds with $\mathbf{u}_{+}=\mathbf{u}$.

First, we define the subsonic set

$$
\mathcal{S}^{\operatorname{sub}}\left(\mathbf{u}_{-}, \alpha_{1}\right)=\left\{\rho_{1}>0 \quad \text { such that } \quad\left(\frac{m}{\alpha_{1} \rho_{1} c_{1}\left(\rho_{1}\right)}\right)^{2}<1\right\}
$$

In order to justify the terminology subsonic, let us underline that for all $\rho_{1}>0$ the quantity

$$
\left(\frac{m}{\alpha_{1} \rho_{1} c_{1}\left(\rho_{1}\right)}\right)
$$

can actually be seen as the ratio of the velocity of fluid 1 in the frame of the linearly degenerate wave of speed $u_{2}: u_{1}-u_{2}$ (by definition $m=\alpha_{1} \rho_{1}\left(u_{1}-u_{2}\right)$ ) and the sound speed $c_{1}\left(\rho_{1}\right)$. We are now in position to prove the following result.

Theorem 2.3. Let $\mathbf{u}_{-}$be a constant state in $\Omega$ and $\alpha_{1+}$ a constant void fraction in $(0,1)$. Assume that $\mathbf{u}_{-}$ is a subsonic state in the sense that $\rho_{1-}$ belongs to $\mathcal{S}^{\mathrm{sub}}\left(\mathbf{u}_{-}, \alpha_{1+}\right)$. Assume moreover that the pressure law $p_{1}$ fulfills

$$
\left\{\begin{array}{l}
p_{1}\left(\rho_{1}\right)>0, \quad p_{1}^{\prime}\left(\rho_{1}\right)>0, \quad p_{1}^{\prime \prime}\left(\rho_{1}\right)>0 \quad \text { for all } \rho_{1}>0 \\
\lim _{\rho_{1} \rightarrow 0} p_{1}\left(\rho_{1}\right)=\lim _{\rho_{1} \rightarrow 0} p_{1}^{\prime}\left(\rho_{1}\right)=0 \\
\lim _{\rho_{1} \rightarrow \infty} p_{1}\left(\rho_{1}\right)=\lim _{\rho_{1} \rightarrow \infty} p_{1}^{\prime}\left(\rho_{1}\right)=+\infty
\end{array}\right.
$$

Then, provided that $\left|\alpha_{1+}-\alpha_{1-}\right|$ is sufficiently small, there exists a unique state $\mathbf{u}_{+}=\mathbf{u}_{+}\left(\mathbf{u}_{-}, \alpha_{1+}\right) \in \Omega$ such that $\rho_{1+} \in \mathcal{S}^{\mathrm{sub}}\left(\mathbf{u}_{-}, \alpha_{1+}\right)$ and the jump relations (2.2) are satisfied.

The proof of this result is classical and is given, for instance, in [19].

It is important to notice that the set of equations (2.2) is nonlinear, and subject to a proximity assumption on the void fractions to be well posed. The next section shows how to deal easily with the nonlinearities by introducing a relaxation approximation of (1.2).

\section{Relaxation approximation}

The aim of this section is to propose a suitable relaxation approximation of system (1.2). For that, we start from the fact that the nonlinearities of (2.2) are closely related to the nonlinearities of the pressure laws $\rho_{k} \mapsto p_{k}\left(\rho_{k}\right)$. In the spirit of $[12,15,26]$, we then consider an enlarged system with two additional scalar unknown quantities associated with two linearizations $\Pi_{k}$ of the pressure laws $p_{k}$. The point is to modify the pressure laws in the convective part of (1.2) in order to get a quasilinear enlarged system, and to move their main nonlinearities in a stiff relaxation term. This relaxation procedure is based on the idea that solutions of the original system (1.2) are formally recovered as the limit of the solutions of the proposed enlarged system with relaxation source term in the regime of an infinite relaxation coefficient (see for instance [13]).

As a relaxation approximation of (1.2), we propose the following system of balance laws:

$$
\partial_{t} \mathbf{v}^{\varepsilon}+\partial_{x} \mathbf{g}^{\varepsilon}\left(\mathbf{v}^{\varepsilon}\right)+\mathbf{d}^{\varepsilon}\left(\mathbf{v}^{\varepsilon}\right) \partial_{x} \mathbf{v}^{\varepsilon}=\frac{1}{\varepsilon} \mathcal{R}^{\varepsilon}\left(\mathbf{v}^{\varepsilon}\right), \quad t>0, \quad x \in \mathbb{R}
$$


where, by omitting the superscripts $\varepsilon$ to ease notations, the unknown vector reads

$$
\mathbf{v}=\left(\begin{array}{c}
\alpha_{1} \\
\mathbf{u}_{1} \\
\mathbf{u}_{2} \\
\alpha_{1} \rho_{1} \mathcal{T}_{1} \\
\alpha_{2} \rho_{2} \mathcal{T}_{2}
\end{array}\right), \quad \text { with } \quad \mathbf{u}_{1}=\left(\begin{array}{c}
\alpha_{1} \rho_{1} \\
\alpha_{1} \rho_{1} u_{1}
\end{array}\right), \quad \mathbf{u}_{2}=\left(\begin{array}{c}
\alpha_{2} \rho_{2} \\
\alpha_{2} \rho_{2} u_{2}
\end{array}\right)
$$

whereas the functions $\mathbf{g}$ and $\mathbf{d}$ are such that

$$
\mathbf{g}(\mathbf{v})=\left(\begin{array}{c}
0 \\
\alpha_{1} \rho_{1} u_{1} \\
\alpha_{1} \rho_{1} u_{1}^{2}+\alpha_{1} \Pi_{1} \\
\alpha_{2} \rho_{2} u_{2} \\
\alpha_{2} \rho_{2} u_{2}^{2}+\alpha_{2} \Pi_{2} \\
\alpha_{1} \rho_{1} \mathcal{T}_{1} u_{1} \\
\alpha_{2} \rho_{2} \mathcal{T}_{2} u_{2}
\end{array}\right), \quad \mathbf{d}(\mathbf{v}) \partial_{x} \mathbf{v}=\left(\begin{array}{c}
u_{I} \partial_{x} \alpha_{1} \\
0 \\
-\Pi_{I} \partial_{x} \alpha_{1} \\
0 \\
\Pi_{I} \partial_{x} \alpha_{1} \\
0 \\
0
\end{array}\right)
$$

The relaxation term $\mathcal{R}(\mathbf{v})$ is defined by

$$
\mathcal{R}(\mathbf{v})=\left(\begin{array}{c}
0 \\
0 \\
0 \\
0 \\
0 \\
\alpha_{1} \rho_{1}\left(\tau_{1}-\mathcal{T}_{1}\right) \\
\alpha_{2} \rho_{2}\left(\tau_{2}-\mathcal{T}_{2}\right)
\end{array}\right)
$$

Here, $\mathcal{T}_{1}$ and $\Pi_{1}$ (respectively $\mathcal{T}_{2}$ and $\Pi_{2}$ ) can be understood as relaxations associated with the specific volume $\tau_{1}=1 / \rho_{1}$ (respectively $\left.\tau_{2}=1 / \rho_{2}\right)$ and the pressure $p_{1}$ (respectively $\left.p_{2}\right)$, and we set

$$
\Pi_{k}=p_{k}\left(1 / \mathcal{T}_{k}\right)+a_{k}^{2}\left(\mathcal{T}_{k}-\tau_{k}\right), \quad k=1,2,
$$

for some constants $a_{1}$ and $a_{2}$ precised just below. These relaxation quantities $\mathcal{T}_{k}$ and $\Pi_{k}$ are expected to converge towards the corresponding equilibrium ones $\tau_{k}$ and $p_{k}$ in the regime of an infinite relaxation rate $\lambda$ $(\lambda \rightarrow \infty)$. However, in order to prevent the relaxation system (3.1) from instabilities in the regime of large values of $\lambda(\lambda \gg 1)$, the free parameters $a_{k}$ must be such that

$$
a_{k}>\rho_{k} c_{k}\left(\rho_{k}\right), \quad k=1,2
$$

for all the $\rho_{k}$ under consideration. This condition is the so-called Whitham condition (see for instance [8,12] and the references therein). We also refer the reader to [7,9] for another relaxation approximation of system (1.2).

Of course, the proposed interfacial velocity $u_{I}$ and pressure $\Pi_{I}$ are naturally defined when setting

$$
u_{I}=u_{2}, \quad \Pi_{I}=\Pi_{1}
$$

in perfect analogy with the choice (1.4) for system (1.2). 
The main interest of this relaxation system lies in the linear degeneracy property of its characteristic fields, unlike system (1.2), see Proposition 1.1. More precisely, the following statement holds.

Proposition 3.1. For all vector $\mathbf{v}$ in the phase space $\Omega^{r}$ defined by

$$
\begin{array}{r}
\Omega^{r}=\left\{\left(\alpha_{1}, \alpha_{1} \rho_{1}, \alpha_{1} \rho_{1} u_{1}, \alpha_{2} \rho_{2}, \alpha_{2} \rho_{2} u_{2}, \alpha_{1} \rho_{1} \mathcal{T}_{1}, \alpha_{2} \rho_{2} \mathcal{T}_{2}\right)^{t} \in \mathbb{R}^{7} \quad\right. \text { such that } \\
\left.0<\alpha_{k}<1 \quad \text { and } \rho_{k}>0 \quad \text { and } \mathcal{T}_{k}>0 \quad \text { for } \quad k=1,2\right\}
\end{array}
$$

the first order system extracted from (3.1), namely

$$
\partial_{t} \mathbf{v}+\partial_{x} \mathbf{g}(\mathbf{v})+\mathbf{d}(\mathbf{v}) \partial_{x} \mathbf{v}=0
$$

admits the following six real eigenvalues

$$
\begin{gathered}
\lambda_{0}^{r}(\mathbf{v})=u_{2}, \\
\lambda_{1}^{r}(\mathbf{v})=u_{1}-a_{1} \tau_{1}, \quad \lambda_{2}^{r}(\mathbf{v})=u_{1}+a_{1} \tau_{1}, \\
\lambda_{3}^{r}(\mathbf{v})=u_{2}-a_{2} \tau_{2}, \quad \lambda_{4}^{r}(\mathbf{v})=u_{2}+a_{2} \tau_{2}, \\
\lambda_{5}^{r}(\mathbf{v})=u_{1},
\end{gathered}
$$

and is hyperbolic (the corresponding right eigenvectors span $\mathbb{R}^{7}$ ) over $\Omega^{r}$ as soon as $u_{2} \neq u_{1} \pm a_{1} \tau_{1}$. Moreover, all the characteristic fields associated with $\left\{\lambda_{i}^{r}\right\}_{i=1, \ldots, 5}$ are linearly degenerate.

Proof. We only propose a sketch of the proof of this result which follows from cumbersome but not difficult calculations.

First, and unlike Proposition 1.1, we found more pleasant to recast the first order system extracted from (3.1) when considering the new vector $\mathbf{w}$ defined by $\mathbf{w}=\left(\alpha_{1}, \rho_{1}, u_{1}, \rho_{2}, u_{2}, \Pi_{1}, \Pi_{2}\right)$ as a main unknown. We then arrive at

$$
\partial_{t} \mathbf{w}+\mathbf{B}(\mathbf{w}) \partial_{x} \mathbf{w}=0
$$

with

$$
\mathbf{B}(\mathbf{w})=\left(\begin{array}{ccccccc}
u_{2} & 0 & 0 & 0 & 0 & 0 & 0 \\
\frac{\rho_{1} u_{1}-\rho_{1} u_{2}}{\alpha_{1}} & u_{1} & \rho_{1} & 0 & 0 & 0 & 0 \\
\frac{\Pi_{1}-\Pi_{I}}{\alpha_{1} \rho_{1}} & 0 & u_{1} & 0 & 0 & \frac{1}{\rho_{1}} & 0 \\
\frac{\rho_{2} u_{2}-\rho_{2} u_{2}}{\alpha_{2}} & 0 & 0 & u_{2} & \rho_{2} & 0 & 0 \\
\frac{\Pi_{2}-\Pi_{I}}{\alpha_{2} \rho_{2}} & 0 & 0 & 0 & u_{2} & 0 & \frac{1}{\rho_{2}} \\
\frac{a_{1}^{2}\left(u_{1}-u_{2}\right)}{\alpha_{1} \rho_{1}} & 0 & \frac{a_{1}^{2}}{\rho_{1}} & 0 & 0 & u_{1} & 0 \\
\frac{a_{2}^{2}\left(u_{2}-u_{2}\right)}{\alpha_{2} \rho_{2}} & 0 & 0 & 0 & \frac{a_{2}^{2}}{\rho_{2}} & 0 & u_{2}
\end{array}\right)
$$

Easy calculations allow to obtain the characteristic polynomial $\mathcal{P}(\mu)$ of this square matrix:

$$
\mathcal{P}(\mu)=\left(u_{1}-\mu\right)\left(u_{2}-\mu\right)^{2}\left(\left(u_{2}-\mu\right)^{2}-\frac{a_{2}^{2}}{\rho_{2}^{2}}\right)\left(\left(u_{1}-\mu\right)^{2}-\frac{a_{1}^{2}}{\rho_{1}^{2}}\right),
$$




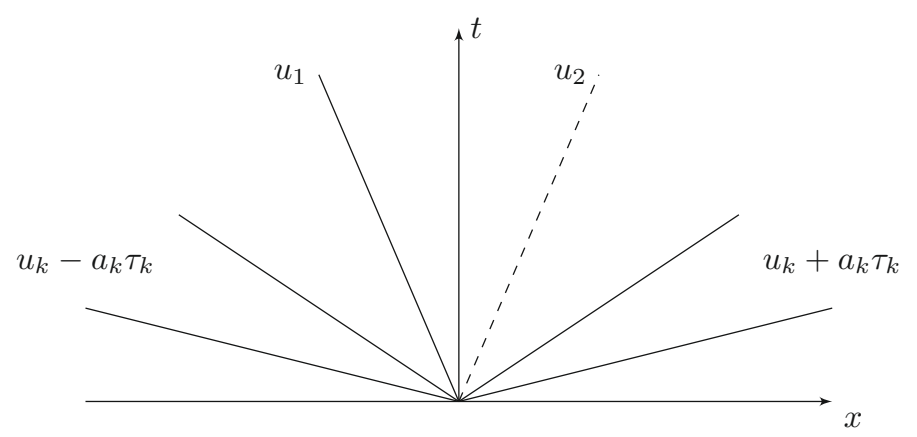

FiguRE 2. General structure in the subsonic regime of the Riemann solution associated with (3.5).

and then the expected eigenvalues $\lambda_{k}^{r}, k=0, \ldots, 5$ in (3.6). Note that the multiplicity of $\lambda_{0}^{r}$ is now two. Provided that $u_{2} \neq \lambda_{k}^{r}, k=1,2$ the following matrix of the associated right eigenvectors is well-defined and invertible

$$
\mathbf{R}(\mathbf{w})=\left(\begin{array}{ccccccc}
1 & 0 & 0 & 0 & 0 & 0 & 0 \\
-\frac{\rho_{1}\left(\lambda_{5}^{r}-\lambda_{k}^{0}\right)^{2}}{\alpha_{1}\left(\lambda_{0}^{r}-\lambda_{1}^{r}\right)\left(\lambda_{0}^{r}-\lambda_{2}^{r}\right)} & 0 & -\frac{1}{a_{1} \tau_{1}^{2}} & \frac{1}{a_{1} \tau_{1}^{2}} & 0 & 0 & 1 \\
\frac{a_{1}^{2} \tau_{1}^{2}\left(\lambda_{5}^{r}-\lambda_{k}^{0}\right)}{\alpha_{1}\left(\lambda_{0}^{r}-\lambda_{1}^{r}\right)\left(\lambda_{0}^{r}-\lambda_{2}^{r}\right)} & 0 & 1 & 1 & 0 & 0 & 0 \\
0 & 1 & 0 & 0 & -\frac{1}{a_{2} \tau_{2}^{2}} & \frac{1}{a_{2} \tau_{2}^{2}} & 0 \\
0 & 0 & 0 & 0 & 1 & 1 & 0 \\
-\frac{a_{1}^{2}\left(\lambda_{5}^{r}-\lambda_{k}^{0}\right)^{2}}{\alpha_{1} \rho_{1}\left(\lambda_{0}^{r}-\lambda_{1}^{r}\right)\left(\lambda_{0}^{r}-\lambda_{2}^{r}\right)} & 0 & -a_{1} & a_{1} & 0 & 0 & 0 \\
\frac{\Pi_{1}-\Pi_{2}}{\alpha_{2}} & 0 & 0 & 0 & -a_{2} & a_{2} & 0
\end{array}\right) .
$$

It is then a classical matter to see that all the characteristic fields are in this case linearly degenerate.

We note that the convective system associated with (3.1) admits one more characteristic speed $u_{1}$ in comparison with (1.2) (see Prop. 1.1). This one is clearly associated with the proposed equation for $\mathcal{T}_{1}$. In addition, the characteristic speed $u_{2}$ is of course still present but now gets a second order multiplicity due to the equation for $\mathcal{T}_{2}$. The other speeds $\lambda_{k}^{r}, k=1, \ldots, 4$ are nothing but linearizations of $\lambda_{k}, k=1, \ldots, 4\left(a_{1}\right.$ and $a_{2}$ have the dimension of two Lagrangian sound speeds). See Figure 2.

\section{Jump RELATIONS ACROSS A CONTACT DisCONTINUITY FOR THE RELAXATION SYSTEM}

In spite of the fact that all the characteristic fields associated with (3.5) are linearly degenerate, it turns out that the corresponding Riemann problem is rather involved, as shown in [19]. Generally speaking, the consequence is that the Riemann solution associated with (3.5) is not given by an explicit formula.

As in Section 2, our objective here is then to characterize the admissible contact discontinuities associated with $\lambda_{0}=u_{2}$ and separating two constant states $\mathbf{v}_{-}$and $\mathbf{v}_{+}$in $\Omega^{r}$. We follow the same procedure. Namely, we first complete the four conservation laws already present in (3.1) by deriving two additional partial differential 
equations satisfied by the smooth solutions of system (3.1). Then, by means of the usual Rankine-Hugoniot jump relations, we propose a full set of conditions for such a solution to be admissible.

Proposition 4.1. Smooth solutions of (3.5) obey the following two partial differential equations

$$
\left\{\begin{array}{l}
\partial_{t}\left(\alpha_{1} \rho_{1} u_{1}+\alpha_{2} \rho_{2} u_{2}\right)+\partial_{x}\left(\alpha_{1} \rho_{1} u_{1}^{2}+\alpha_{2} \rho_{2} u_{2}^{2}+\alpha_{1} \Pi_{1}+\alpha_{2} \Pi_{2}\right)=0 \\
\partial_{t} u_{1}+\partial_{x} \frac{u_{1}^{2}-a_{1}^{2} \tau_{1}^{2}}{2}+\tau_{1} \partial_{x} I_{1}=0
\end{array}\right.
$$

where we have set $I_{1}=p_{1}\left(1 / \mathcal{T}_{1}\right)+a_{1}^{2} \mathcal{T}_{1}$.

Proof. The proposed equations follow from classical manipulations on system (3.5).

We first notice that among these two additional equations, only one is under conservation form. However, we will see in the following statement that $\mathcal{T}_{1}$ (and thus $I_{1}$ ) is seen to be constant across the contact associated with $\lambda_{0}$ (in the absence of resonance with $u_{1}$ ). Therefore the non conservative product $\tau_{1} \partial_{x} I_{1}$ cancels and the second equation actually provides an additional conservation law. We then easily get the following definition, comparable with Definition 2.2.

Definition 4.2. Let $\mathbf{v}_{-}$and $\mathbf{v}_{+}$be two constant states in $\Omega^{r}$. These states can be joined by an admissible $\lambda_{0}$ contact discontinuity if and only if the following jump relations hold:

$$
\left\{\begin{array}{c}
u_{2}:=u_{2-}=u_{2+}, \\
m:=\alpha_{1-} \rho_{1-}\left(u_{1-}-u_{2}\right)=\alpha_{1+} \rho_{1+}\left(u_{1+}-u_{2}\right), \\
m u_{1-}+\alpha_{1-} \Pi_{1-}+\alpha_{2-} \Pi_{2-}=m u_{1+}+\alpha_{1+} \Pi_{1+}+\alpha_{2+} \Pi_{2+}, \\
m \mathcal{T}_{1-}=m \mathcal{T}_{1+}, \\
\frac{m^{2}}{2 \alpha_{1-}^{2} \rho_{1-}^{2}}-a_{1}^{2} \frac{\tau_{1-}^{2}}{2}=\frac{m^{2}}{2 \alpha_{1+}^{2} \rho_{1+}^{2}}-a_{1}^{2} \frac{\tau_{1+}^{2}}{2}
\end{array}\right.
$$

In such a situation, the speed of propagation of the discontinuity is given by $u_{2}$.

It is worth noticing that the first two equations in (4.1) and (2.2) are identical, while the third are similar in the sense that $\Pi_{1}$ and $\Pi_{2}$ play the part of linearized pressure laws. On the contrary, and thanks to the proposed relaxation approximation, the last equation in (4.1) is significantly simplified (i.e. less non linear) in comparison to the last equation in (2.2). We seek now to obtain a result analogous to Theorem 2.3.

Theorem 4.3. Let $\mathbf{v}_{-}$be a constant state in $\Omega^{r}$ and $\alpha_{1+}$ a constant void fraction in $(0,1)$. Assume that $\mathbf{v}_{-}$is a subsonic state in the sense that

$$
\left(\frac{m}{\alpha_{1+} a_{1}}\right)^{2}<1
$$

Then, provided that $\left|\alpha_{1+}-\alpha_{1-}\right|$ is sufficiently small, there exists a unique subsonic point $\rho_{1+}$ such that the last equation in (4.1) holds true. It is explicitly given by

$$
\rho_{1+}=\sqrt{\frac{\frac{m}{\alpha_{1+a_{1}}}-1}{\frac{m}{\alpha_{1-} a_{1}}-1}} \rho_{1-} .
$$

Moreover, if we get for any given $\mathcal{T}_{2+}$

$$
-m\left(u_{1+}-u_{1-}\right)+\left(\alpha_{1-} \Pi_{1-}-\alpha_{1+} \Pi_{1+}\right)+\alpha_{2-} \Pi_{2-}-\alpha_{2+} I_{2+}<0
$$


with $I_{2}=p_{2}\left(1 / \mathcal{T}_{2}\right)+a_{2} \mathcal{T}_{2}$, and $u_{1+}$ defined by the second equation in $(4.1)$, then we can define uniquely $\rho_{2+}>0$ such that the third equation in (4.1) holds true. It is explicitly given by

$$
\tau_{2+}=\frac{1}{\rho_{2+}}=-\frac{-m\left(u_{1+}-u_{1-}\right)+\left(\alpha_{1-} \Pi_{1-}-\alpha_{1+} \Pi_{1+}\right)+\alpha_{2-} \Pi_{2-}-\alpha_{2+} I_{2+}}{\alpha_{2+} a_{2}^{2}}
$$

and we thus can find a unique state $\mathbf{v}_{+}=\mathbf{v}_{+}\left(\mathbf{v}_{-}, \alpha_{1+}\right) \in \Omega^{r}$ so that the jump relations (4.1) are satisfied.

Proof. This theorem is not difficult to establish thanks to the linearized formulas (4.1). First of all, the state $\mathbf{v}_{-}$is assumed to be subsonic in the sense of inequality (4.2), which is equivalent to

$$
-1<\frac{m}{\alpha_{1+a_{1}}}<1
$$

Provided that $\left|\alpha_{1+}-\alpha_{1-}\right|$ is sufficiently small, we also have

$$
-1<\frac{m}{\alpha_{1-} a_{1}}<1
$$

Then, it is easily checked that the unique solution $\rho_{1+}>0$ of the last equation in (4.1) is given by the welldefined formula (4.3). Defining $u_{2}=u_{2+}=u_{2-}$ and $u_{1+}=u_{2}+m / \alpha_{1+} \rho_{1+}$ according to the first two equations in (4.1), it is easy to see that under (4.4) the unique solution $\rho_{2+}>0$ of the third equation in (4.1) is explicitly given by (4.5).

Remark 4.4. In the context of the proposed relaxation approximation, $\rho_{2+}$ is now explicitly given.

\section{NuMERICAL APPROXIMATION}

In this section, we propose to derive a numerical scheme for approximating the weak solutions of the following initial value problem associated with system (1.2):

$$
\left\{\begin{array}{l}
\partial_{t} \mathbf{u}+\partial_{x} \mathbf{f}(\mathbf{u})+\mathbf{c}(\mathbf{u}) \partial_{x} \mathbf{u}=0, \quad t>0, \quad x \in \mathbb{R} \\
\mathbf{u}(x, 0)=\mathbf{u}_{0}(x)
\end{array}\right.
$$

Of course, the proposed relaxation system (3.1) is going to be used. Let us first introduce some notations. In what follows, $\Delta t$ and $\Delta x$ are respectively the time and space steps that we assume to be constant for simplicity. We also set $\nu=\frac{\Delta t}{\Delta x}$ and define both the mesh interfaces $x_{j+1 / 2}=j \Delta x$ for $j \in \mathbb{Z}$, and the intermediate times $t^{n}=n \Delta t$ for $n \in \mathbb{N}$. As usual in the context of finite volume methods, the approximate solution $x \mapsto \mathbf{u}_{\nu}\left(x, t^{n}\right)$ is assumed to be constant at each time $t^{n}$ in cells $\mathcal{C}_{j}=\left[x_{j-1 / 2}, x_{j+1 / 2}\right.$ [ and the corresponding values are called $\mathbf{u}_{j}^{n}$. Therefore, we have

$$
\mathbf{u}_{\nu}\left(x, t^{n}\right)=\mathbf{u}_{j}^{n} \quad \text { for all } \quad x \in \mathcal{C}_{j}, \quad j \in \mathbb{Z}, \quad n \in \mathbb{N} .
$$

At time $t=0$, we use the initial condition $\mathbf{u}_{0}$ provided in (5.1) to define the sequence $\left(\mathbf{u}_{j}^{0}\right)_{j}$ as follows:

$$
\mathbf{u}_{j}^{0}=\frac{1}{\Delta x} \int_{x_{j-1 / 2}}^{x_{j+1 / 2}} \mathbf{u}_{0}(x) \mathrm{d} x, \quad \forall j \in \mathbb{Z} .
$$

\subsection{The relaxation method}

Let us now briefly describe the two-step splitting technique associated with the relaxation system (3.1) in order to define $\mathbf{u}_{\nu}\left(., t^{n+1}\right)$ from $\mathbf{u}_{\nu}\left(., t^{n}\right)$. Such a strategy is very classical in the context of relaxation methods 
and proposes to first treat the convective part of (3.1), and then to take into account the relaxation source term. We first introduce the piecewise constant approximate solution $x \mapsto \mathbf{v}_{\nu}\left(x, t^{n}\right)$ at each time $t^{n}$ of system (3.1)

$$
\mathbf{v}_{j}^{n}=\left(\begin{array}{c}
\mathbf{u}_{j}^{n} \\
\left(\alpha_{1} \rho_{1} \mathcal{T}_{1}\right)_{j}^{n} \\
\left(\alpha_{2} \rho_{2} \mathcal{T}_{2}\right)_{j}^{n}
\end{array}\right)
$$

At time $t=0$, function $x \mapsto \mathbf{v}_{\nu}\left(x, t^{0}\right)$ is set at equilibrium, which means

$$
\left(\alpha_{1} \rho_{1} \mathcal{T}_{1}\right)_{j}^{0}=\left(\alpha_{1}\right)_{j}^{0} \quad \text { and } \quad\left(\alpha_{2} \rho_{2} \mathcal{T}_{2}\right)_{j}^{0}=\left(\alpha_{2}\right)_{j}^{0}
$$

The two steps are defined as follows.

Step 1. Evolution in time $\left(t^{n} \rightarrow t^{n+1-}\right)$

In this first step, the following Cauchy problem is numerically solved for small times $t \in[0, \Delta t]$ :

$$
\left\{\begin{array}{l}
\partial_{t} \mathbf{v}+\partial_{x} \mathbf{g}(\mathbf{v})+\mathbf{d}(\mathbf{v}) \partial_{x} \mathbf{v}=0, \quad x \in \mathbb{R} \\
\mathbf{v}(x, 0)=\mathbf{v}_{\nu}\left(x, t^{n}\right)
\end{array}\right.
$$

Since $x \mapsto \mathbf{v}_{\nu}\left(x, t^{n}\right)$ is piecewise constant, the exact solution of (5.2) is theoretically obtained by gluing together the solutions of the Riemann problems set at each interface $x_{j+1 / 2}$ provided that $\Delta t$ is such that

$$
\frac{\Delta t}{\Delta x} \max _{\mathbf{v}}\left\{\left|\lambda_{i}^{r}(\mathbf{v})\right|, \quad i=0, \ldots, 5\right\} \leq \frac{1}{2}
$$

for all the $\mathbf{v}$ under consideration. More precisely

$$
\mathbf{v}_{\nu}(x, t)=\mathbf{v}_{\mathbf{r}}\left(\frac{x-x_{j+1 / 2}}{t} ; \mathbf{v}_{j}^{n}, \mathbf{v}_{j+1}^{n}\right) \quad \text { for all }(x, t) \in\left[x_{j}, x_{j+1}\right] \times[0, \Delta t],
$$

where $(x, t) \mapsto \mathbf{v}_{\mathbf{r}}\left(\frac{x}{t} ; \mathbf{v}_{L}, \mathbf{v}_{R}\right)$ denotes the exact self-similar solution of the Riemann problem

$$
\left\{\begin{array}{l}
\partial_{t} \mathbf{v}+\partial_{x} \mathbf{g}(\mathbf{v})+\mathbf{d}(\mathbf{v}) \partial_{x} \mathbf{v}=0, \quad x \in \mathbb{R}, \quad t \in \mathbb{R}^{+, \star} \\
\mathbf{v}(x, 0)= \begin{cases}\mathbf{v}_{L} & \text { if } \quad x<0, \\
\mathbf{v}_{R} & \text { if } \quad x>0,\end{cases}
\end{array}\right.
$$

for $\mathbf{v}_{L}$ and $\mathbf{v}_{R}$ given in the phase space $\Omega^{r}$.

Note that these exact Riemann solutions are difficult to calculate as already explained at the beginning of Section 4. Therefore some approximate Riemann solutions $(x, t) \mapsto \tilde{\mathbf{v}}_{\mathbf{r}}\left(\frac{x}{t} ; \mathbf{v}_{L}, \mathbf{v}_{R}\right)$ of $(5.5)$ are expected to be used in practice, giving rise to a natural approximate solution $(x, t) \mapsto \tilde{\mathbf{v}}_{\nu}(x, t)$ of $(5.2)$. This issue is going to be discussed in detail in the next subsection.

In order to define a piecewise constant approximate solution on each cell $\mathcal{C}_{j}$ at time $t^{n+1-}$, we then propose the following classical averaging procedure of $x \mapsto \tilde{\mathbf{v}}_{\nu}(x, \Delta t)$ :

$$
\mathbf{v}_{j}^{n+1-}=\left(\begin{array}{c}
\mathbf{u}_{j}^{n+1-} \\
\left(\alpha_{1} \rho_{1} \mathcal{T}_{1}\right)_{j}^{n+1-} \\
\left(\alpha_{2} \rho_{2} \mathcal{T}_{2}\right)_{j}^{n+1-}
\end{array}\right)=\frac{1}{\Delta x} \int_{x_{j-1 / 2}}^{x_{j+1 / 2}} \tilde{\mathbf{v}}_{\nu}(x, \Delta t) \mathrm{d} x, \quad j \in \mathbb{Z} .
$$


Step 2. Relaxation $\left(t^{n+1-} \rightarrow t^{n+1}\right)$

In this second step, we propose to solve at time $t^{n}+\Delta t$ the ordinary differential equations

$$
\partial_{t} \mathbf{v}^{\lambda}=\lambda \mathcal{R}\left(\mathbf{v}^{\lambda}\right), \quad x \in \mathbb{R}
$$

in the asymptotic regime $\lambda \rightarrow \infty$. As initial condition, we take the function $x \mapsto \mathbf{v}_{\nu}\left(x, t^{n+1-}\right)$ obtained at the end of the first step and defined by

$$
\mathbf{v}_{\nu}\left(x, t^{n+1-}\right)=\mathbf{v}_{j}^{n+1-} \quad \text { for all } \quad x \in \mathcal{C}_{j}, \quad j \in \mathbb{Z} .
$$

Using the definition (3.3) of the relaxation source term $\mathcal{R}$, this clearly amounts to set

$$
\mathbf{v}_{j}^{n+1}=\left(\begin{array}{c}
\mathbf{u}_{j}^{n+1} \\
\left(\alpha_{1} \rho_{1} \mathcal{T}_{1}\right)_{j}^{n+1} \\
\left(\alpha_{2} \rho_{2} \mathcal{T}_{2}\right)_{j}^{n+1}
\end{array}\right)
$$

with $\mathbf{u}_{j}^{n+1}=\mathbf{u}_{j}^{n+1-}$ and $\left(\mathcal{T}_{k}\right)_{j}^{n+1}=\left(\tau_{k}\right)_{j}^{n+1}$ i.e.

$$
\left(\alpha_{1} \rho_{1} \mathcal{T}_{1}\right)_{j}^{n+1}=\left(\alpha_{1}\right)_{j}^{n+1-}, \quad\left(\alpha_{2} \rho_{2} \mathcal{T}_{2}\right)_{j}^{n+1}=\left(\alpha_{2}\right)_{j}^{n+1-}
$$

This completes the description of the two-step relaxation method.

\subsection{The approximate Riemann solution $(x, t) \mapsto \tilde{\mathbf{v}}_{\mathbf{r}}\left(\frac{x}{t} ; \mathbf{v}_{L}, \mathbf{v}_{R}\right)$}

Our objective in this section is to detail the Riemann solver $(x, t) \mapsto \tilde{\mathbf{v}}_{\mathbf{r}}\left(\frac{x}{t} ; \mathbf{v}_{L}, \mathbf{v}_{R}\right)$ associated with (5.5) for any given $\mathbf{v}_{L}$ and $\mathbf{v}_{R}$ in the phase space $\Omega^{r}$, and implicitly used in the update formula (5.6). According to the previous subsection, the initial states $\mathbf{v}_{L}$ and $\mathbf{v}_{R}$ are assumed to be at equilibrium, i.e. such that

$$
\mathbf{v}_{L, R}=\left(\begin{array}{c}
\mathbf{u}_{L, R} \\
\left(\alpha_{1} \rho_{1} \mathcal{T}_{1}\right)_{L, R} \\
\left(\alpha_{2} \rho_{2} \mathcal{T}_{2}\right)_{L, R}
\end{array}\right)=\left(\begin{array}{c}
\mathbf{u}_{L, R} \\
\alpha_{1 L, R} \\
\alpha_{2 L, R}
\end{array}\right)
$$

with $\mathbf{u}_{L}$ and $\mathbf{u}_{R}$ in $\Omega$.

As already discussed in Section 4, the exact Riemann solution to (5.5) is not explicitly known given two different void fractions $\alpha_{1 L}$ and $\alpha_{1 R}$. To lessen computational costs in the numerical procedure, we then decide not to compute it exactly but to use a suitable approximate Riemann solution. In fact, the proposed approximate Riemann solver turns out to be an exact Riemann solver but associated with a simpler system.

Let us briefly discuss the derivation of such a system. We have observed in the previous paragraph that (5.5) is easy to deal with when $\alpha_{1 L}=\alpha_{1 R}$. The reason is that in this very particular case, the non conservative product $\Pi_{I} \partial_{x} \alpha_{1}$ is known a priori (is zero in fact) and, in some sense, no longer enters the resolution of the Riemann problem. Recall that this nonconservative product acts on the contact only, while its $\operatorname{mass}_{2}\left\langle\Pi_{I} \partial_{x} \alpha_{1}\right\rangle$ represents (to within a sign) the jump of $\alpha_{2} \Pi_{2}$ between the right and left states of the discontinuity ${ }^{6}$. Indeed, if $u_{2}^{*}$ denotes the speed of propagation of the contact, the Rankine-Hugoniot jump relations applied to the fifth equation in (3.1) (with $\lambda=0$ ) give

$$
-u_{2}^{*}\left[\alpha_{2} \rho_{2} u_{2}\right]+\left[\alpha_{2} \rho_{2} u_{2}^{2}+\alpha_{2} \Pi_{2}\right]+\left\langle\Pi_{I} \partial_{x} \alpha_{1}\right\rangle=0,
$$

that is (recall that $u_{2}$ is continuous across the contact and equals $u_{2}^{*}$ )

$$
\left\langle\Pi_{I} \partial_{x} \alpha_{1}\right\rangle=-\left[\alpha_{2} \Pi_{2}\right] .
$$

\footnotetext{
${ }^{6}$ The jump of a quantity $X$ between the right and left states of a discontinuity is noted $[X]$ in the following.
} 
The value $\left[\alpha_{2} \Pi_{2}\right]$ of this jump depends on the left and right initial states $\mathbf{v}_{L}$ and $\mathbf{v}_{R}$ but note that by the equilibrium property (5.8), it can also be understood as a given function of $\mathbf{u}_{L}$ and $\mathbf{u}_{R}$. We thus have:

$$
\Pi_{I} \partial_{x} \alpha_{1}=-\left[\alpha_{2} \Pi_{2}\right]\left(\mathbf{u}_{L}, \mathbf{u}_{R}\right) \delta_{x-u_{2}^{*} t}
$$

Following the same idea, we propose not to find the exact value $\left[\alpha_{2} \Pi_{2}\right]\left(\mathbf{u}_{L}, \mathbf{u}_{R}\right)$, i.e. not to consider it as an unknown, but to guess it a priori in order to facilitate the resolution of the Riemann problem. In other words, we replace the actual value $\left[\alpha_{2} \Pi_{2}\right]\left(\mathbf{u}_{L}, \mathbf{u}_{R}\right)$ with an estimation $\overline{\left[\alpha_{2} \Pi_{2}\right]}\left(\mathbf{u}_{L}, \mathbf{u}_{R}\right)$ of the jump of $\alpha_{2} \Pi_{2}$ across the contact. This degree of freedom may be put in touch with the relative flexibility in the discretization of nonconservative products associated with linearly degenerate characteristic fields (see for instance [25]).

We are thus led to introduce the following system:

$$
\partial_{t} \tilde{\mathbf{v}}+\partial_{x} \mathbf{g}(\tilde{\mathbf{v}})=-\tilde{\mathbf{d}}(\tilde{\mathbf{v}}) \partial_{x} \tilde{\mathbf{v}}, \quad t>0, \quad x \in \mathbb{R}
$$

with

$$
\tilde{\mathbf{d}}(\tilde{\mathbf{v}}) \partial_{x} \tilde{\mathbf{v}}=\left(\begin{array}{c}
u_{I} \partial_{x} \alpha_{1} \\
0 \\
+\overline{\left[\alpha_{2} \Pi_{2}\right]}\left(\mathbf{u}_{L}, \mathbf{u}_{R}\right) \delta_{x-u_{2}^{*} t} \\
0 \\
-\overline{\left[\alpha_{2} \Pi_{2}\right]}\left(\mathbf{u}_{L}, \mathbf{u}_{R}\right) \delta_{x-u_{2}^{*} t} \\
-m\left(\mathcal{T}_{1 R}-\mathcal{T}_{1 L}\right) \delta_{x-u_{2}^{*} t} \\
0
\end{array}\right) .
$$

The vectors $\tilde{\mathbf{v}}$ and $\mathbf{g}$ are kept unchanged, namely

$$
\tilde{\mathbf{v}}=\left(\begin{array}{c}
\alpha_{1} \\
\mathbf{u}_{1} \\
\mathbf{u}_{2} \\
\alpha_{1} \rho_{1} \mathcal{T}_{1} \\
\alpha_{2} \rho_{2} \mathcal{T}_{2}
\end{array}\right), \quad \mathbf{g}(\tilde{\mathbf{v}})=\left(\begin{array}{c}
0 \\
\alpha_{1} \rho_{1} u_{1} \\
\alpha_{1} \rho_{1} u_{1}^{2}+\alpha_{1} \Pi_{1} \\
\alpha_{2} \rho_{2} u_{2} \\
\alpha_{2} \rho_{2} u_{2}^{2}+\alpha_{2} \Pi_{2} \\
\alpha_{1} \rho_{1} \mathcal{T}_{1} u_{1} \\
\alpha_{2} \rho_{2} \mathcal{T}_{2} u_{2}
\end{array}\right)
$$

On the contrary, we remark from the sixth equation in (5.9) that $\alpha_{1} \rho_{1} \mathcal{T}_{1}$ no longer evolves according to the original conservation law

$$
\partial_{t} \alpha_{1} \rho_{1} \mathcal{T}_{1}+\partial_{x} \alpha_{1} \rho_{1} \mathcal{T}_{1} u_{1}=0
$$

across the contact, which would mean $m\left[\mathcal{T}_{1}\right]=0$ using the notations of Definition 4.2. If the relative mass flux $m \neq 0$, this would imply the continuity of $\mathcal{T}_{1}\left(\left[\mathcal{T}_{1}\right]=0\right)$. Instead, we now impose

$$
\partial_{t} \alpha_{1} \rho_{1} \mathcal{T}_{1}+\partial_{x} \alpha_{1} \rho_{1} \mathcal{T}_{1} u_{1}=m\left(\mathcal{T}_{1 R}-\mathcal{T}_{1 L}\right) \delta_{x-u_{2}^{*} t}
$$

We thus have $\left[\mathcal{T}_{1}\right]=\left(\mathcal{T}_{1 R}-\mathcal{T}_{1 L}\right)$ across the contact. The reason of such a modification is motivated by the validity of the accuracy property stated in Proposition 5.6 and Theorem 5.8 below. Additional explanations will be given in Remark 5.7 (Sect. 5.3). Another objective of this term is to make trivial the $\lambda_{5}^{r}$-discontinuity in the resolution of the Riemann problem (see Thm. 5.4 below). Recall indeed that the eigenvalue $\lambda_{5}^{r}=u_{1}$ is not present in the equilibrium system (1.2) (see Prop. 1.1).

Remark 5.1. The eigenvalues of system (5.9) are the same than the ones of system (3.5). Moreover, all its characteristic fields are linearly degenerate. 
On the $\lambda_{0}$-contact discontinuity, the first two relations in (4.1) still hold ${ }^{7}$ :

$$
\begin{aligned}
u_{2}:=u_{2-} & =u_{2+} \\
m:=\alpha_{1-} \rho_{1-}\left(u_{1-}-u_{2}\right) & =\alpha_{1+} \rho_{1+}\left(u_{1+}-u_{2}\right) .
\end{aligned}
$$

Therefore, the parameter $m$ is well defined in $\tilde{\mathbf{d}}(\tilde{\mathbf{v}})$.

For this section to be completed, it remains now to give both the definition of $\overline{\left[\alpha_{2} \Pi_{2}\right]}\left(\mathbf{u}_{L}, \mathbf{u}_{R}\right)$ and the Riemann solution associated with (5.9). This is the objective of the next two paragraphs.

Estimation of $\overline{\left[\alpha_{2} \Pi_{2}\right]}\left(\mathbf{u}_{L}, \mathbf{u}_{R}\right)$ across the contact. This part is devoted to the definition of $\overline{\left[\alpha_{2} \Pi_{2}\right]}\left(\mathbf{u}_{L}, \mathbf{u}_{R}\right)$. Recall that this quantity aims at providing a relevant approximation of the actual jump $\left[\alpha_{2} \Pi_{2}\right]\left(\mathbf{u}_{L}, \mathbf{u}_{R}\right)$ across the contact in the solution of (5.5). For the sake of accuracy, we would like this approximate value to be exact when $\mathbf{u}_{L}$ and $\mathbf{u}_{R}$ can actually be joined by an admissible contact, i.e. when $\mathbf{u}_{-}=\mathbf{u}_{L}$ and $\mathbf{u}_{+}=\mathbf{u}_{R}$ are such that jump relations (2.2) associated with the equilibrium system (1.2) are satisfied.

With this in mind, we first propose to define from $\mathbf{u}_{L}$ and $\mathbf{u}_{R}$ two reconstructed states $\overline{\mathbf{u}}_{L}$ and $\overline{\mathbf{u}}_{R}$ such that $\overline{\mathbf{u}}_{L}$ and $\mathbf{u}_{R}$ on the one hand and $\mathbf{u}_{L}$ and $\overline{\mathbf{u}}_{R}$ on the other hand can be joined by an admissible contact. In other words, and using the proposed notation in Theorem 2.3, we set

$$
\overline{\mathbf{u}}_{L}=\mathbf{u}_{+}\left(\mathbf{u}_{R}, \alpha_{1 L}\right) \quad \text { and } \quad \overline{\mathbf{u}}_{R}=\mathbf{u}_{+}\left(\mathbf{u}_{L}, \alpha_{1 R}\right)
$$

At this stage, these two admissible contact discontinuities provide us with two possible choices of $\overline{\left[\alpha_{2} \Pi_{2}\right]}\left(\mathbf{u}_{L}, \mathbf{u}_{R}\right)$, namely $\overline{\left[\alpha_{2} \Pi_{2}\right]}\left(\mathbf{u}_{L}, \mathbf{u}_{R}\right)=\left(\alpha_{2} \Pi_{2}\right)\left(\overline{\mathbf{u}}_{R}\right)-\left(\alpha_{2} \Pi_{2}\right)\left(\mathbf{u}_{L}\right)$ and $\overline{\left[\alpha_{2} \Pi_{2}\right]}\left(\mathbf{u}_{L}, \mathbf{u}_{R}\right)=\left(\alpha_{2} \Pi_{2}\right)\left(\mathbf{u}_{R}\right)-\left(\alpha_{2} \Pi_{2}\right)\left(\overline{\mathbf{u}}_{L}\right)$. Of course, we note that if $\mathbf{u}_{L}$ and $\mathbf{u}_{R}$ are joined by an admissible contact, we have $\overline{\mathbf{u}}_{L}=\mathbf{u}_{L}$ and $\overline{\mathbf{u}}_{R}=\mathbf{u}_{R}$ so that both possibilities coincide with the exact jump $\left[\alpha_{2} \Pi_{2}\right]\left(\mathbf{u}_{L}, \mathbf{u}_{R}\right)$. In the general case, we propose the following natural choice

$$
\overline{\left[\alpha_{2} \Pi_{2}\right]}\left(\mathbf{u}_{L}, \mathbf{u}_{R}\right)=\left\{\begin{array}{c}
\left(\alpha_{2} \Pi_{2}\right)\left(\overline{\mathbf{u}}_{R}\right)-\left(\alpha_{2} \Pi_{2}\right)\left(\mathbf{u}_{L}\right) \quad \text { if } \quad d\left(\mathbf{u}_{R}, \overline{\mathbf{u}}_{R}\right) \leq d\left(\overline{\mathbf{u}}_{L}, \mathbf{u}_{L}\right), \\
\left(\alpha_{2} \Pi_{2}\right)\left(\mathbf{u}_{R}\right)-\left(\alpha_{2} \Pi_{2}\right)\left(\overline{\mathbf{u}}_{L}\right) \text { if } d\left(\mathbf{u}_{R}, \overline{\mathbf{u}}_{R}\right)>d\left(\overline{\mathbf{u}}_{L}, \mathbf{u}_{L}\right),
\end{array}\right.
$$

where $d(.,$.$) denotes the Euclidean distance function. Actually this choice criterion doesn't seem to play an$ important role: in the numerical tests we performed, no difference can be spotted if one estimate is preferred to the other (see Fig. 9).

Remark 5.2. In this section $\alpha_{1 L} \neq \alpha_{1 R}$ is assumed. It is however worth noticing from a continuity point of view that if $\alpha_{1 L}=\alpha_{1 R}$ we have by definition $\overline{\mathbf{u}}_{L}=\mathbf{u}_{R}$ and $\overline{\mathbf{u}}_{R}=\mathbf{u}_{L}$, and then $\overline{\left[\alpha_{2} \Pi_{2}\right]}\left(\mathbf{u}_{L}, \mathbf{u}_{R}\right)=0$ as expected.

The Riemann solution $(x, t) \mapsto \tilde{\mathbf{v}}_{\mathbf{r}}\left(\frac{x}{t} ; \mathbf{v}_{L}, \mathbf{v}_{R}\right)$. We now give the Riemann solution $(x, t) \mapsto \tilde{\mathbf{v}}_{\mathbf{r}}\left(\frac{x}{t} ; \mathbf{v}_{L}, \mathbf{v}_{R}\right)$ of

$$
\left\{\begin{array}{l}
\partial_{t} \tilde{\mathbf{v}}+\partial_{x} \mathbf{g}(\tilde{\mathbf{v}})=-\tilde{\mathbf{d}}(\tilde{\mathbf{v}}) \partial_{x} \tilde{\mathbf{v}}, \quad x \in \mathbb{R}, \quad t \in \mathbb{R}^{+, \star} \\
\mathbf{v}(x, 0)=\left\{\begin{array}{lll}
\mathbf{v}_{L} & \text { if } \quad x<0 \\
\mathbf{v}_{R} & \text { if } \quad x>0,
\end{array}\right.
\end{array}\right.
$$

with $\mathbf{v}_{L}$ and $\mathbf{v}_{R}$ in the phase space $\Omega^{r}$.

Before stating the corresponding theorem, let us briefly discuss this issue and more precisely the proposed admissibility criterion for defining the contact discontinuities. Recall that, by Definition 4.2 any admissible contact discontinuity of the original relaxation system (3.5) (with $\lambda=0$ ) should satisfy the five jump relations (4.1). We now deal with the new system (5.9) that proposes to impose two relations across these discontinuities, namely the jumps of $\mathcal{T}_{1}$ and $\alpha_{2} \Pi_{2}$ according to

$$
m\left[\mathcal{T}_{1}\right]=m\left(\mathcal{T}_{1 R}-\mathcal{T}_{1 L}\right) \quad \text { and } \quad\left[\alpha_{2} \Pi_{2}\right]=\overline{\left[\alpha_{2} \Pi_{2}\right]}\left(\mathbf{u}_{L}, \mathbf{u}_{R}\right)
$$

\footnotetext{
${ }^{7}$ The subscripts - and + refer to the states on each side of the discontinuity.
} 
These relations modify the definition of an admissible contact (4.1). We recall that the first two equations in (4.1) still hold. We suggest to keep on imposing the conservation of momentum and then we define:

Definition 5.3. A $\lambda_{0}$ contact discontinuity of the modified relaxation system (5.9), separating two constant states $\mathbf{v}_{-}$and $\mathbf{v}_{+}$such that $\alpha_{1-} \neq \alpha_{1+}$ and propagating with speed $u_{2}$, is said to be admissible if and only if the following jump relations hold:

$$
\left\{\begin{array}{c}
u_{2}:=u_{2-}=u_{2+} \\
m:=\alpha_{1-} \rho_{1-}\left(u_{1-}-u_{2}\right)=\alpha_{1+} \rho_{1+}\left(u_{1+}-u_{2}\right) \\
m u_{1-}+\alpha_{1-} \Pi_{1-}+\alpha_{2-} \Pi_{2-}=m u_{1+}+\alpha_{1+} \Pi_{1+}+\alpha_{2+} \Pi_{2+} \\
m\left(\mathcal{T}_{1+}-\mathcal{T}_{1-}\right)=m\left(\mathcal{T}_{1 R}-\mathcal{T}_{1 L}\right) \\
\alpha_{2+} \Pi_{2+}-\alpha_{2-} \Pi_{2-}=\overline{\left[\alpha_{2} \Pi_{2}\right]}\left(\mathbf{u}_{L}, \mathbf{u}_{R}\right) .
\end{array}\right.
$$

Before stating the main result of this part, we need to precise how to chose the two constants $a_{1}$ and $a_{2}$.

Theorem 5.4. Let $\mathbf{v}_{L}$ and $\mathbf{v}_{R}$ be two constant states in $\Omega^{r}$ at equilibrium (i.e. (5.8) holds true) and such that $\alpha_{1 L} \neq \alpha_{1 R}$. Moreover, let the free parameters $a_{k}, k=1,2$ to be chosen large enough to satisfy the following inequalities on one hand

$$
\left\{\begin{array}{l}
\lambda_{1}^{r}\left(\mathbf{v}_{L}\right)<u_{1-}, \quad u_{2}<\lambda_{2}^{r}\left(\mathbf{v}_{R}\right) \\
\lambda_{1}^{r}\left(\mathbf{v}_{L}\right)<u_{2}, \quad u_{1+}<\lambda_{2}^{r}\left(\mathbf{v}_{R}\right) \\
\lambda_{3}^{r}\left(\mathbf{v}_{L}\right)<u_{2}<\lambda_{4}^{r}\left(\mathbf{v}_{R}\right)
\end{array}\right.
$$

and the following simplified Whitham condition on the other hand

$$
a_{k}\left(\mathbf{u}_{L}, \mathbf{u}_{R}\right)>\max \left(\rho_{k L} c_{k}\left(\rho_{k L}\right), \rho_{k R} c_{k}\left(\rho_{k R}\right)\right), \quad k=1,2 .
$$

Then, the self-similar weak solution $(x, t) \mapsto \tilde{\mathbf{v}}_{\mathbf{r}}\left(x / t, \mathbf{v}_{L}, \mathbf{v}_{R}\right)$ of (5.11) that presents an admissible $\lambda_{0}$ contact discontinuity in the sense of Definition 5.3 is such that

$$
\tilde{\mathbf{v}}_{\mathbf{r}}\left(x / t, \mathbf{v}_{L}, \mathbf{v}_{R}\right)=\left(\begin{array}{c}
\alpha_{1} \\
\mathbf{u}_{1} \\
\mathbf{u}_{2} \\
\alpha_{1} \rho_{1} \mathcal{T}_{1} \\
\alpha_{2} \rho_{2} \mathcal{T}_{2}
\end{array}\right)\left(x / t, \mathbf{v}_{L}, \mathbf{v}_{R}\right)
$$

where first, the main functions $(x, t) \mapsto \mathbf{u}_{1}\left(x / t, \mathbf{v}_{L}, \mathbf{v}_{R}\right)$ and $(x, t) \mapsto \mathbf{u}_{2}\left(x / t, \mathbf{v}_{L}, \mathbf{v}_{R}\right)$ exhibit two intermediate constant states:

and then,

$$
\begin{gathered}
\mathbf{u}_{1}\left(x / t, \mathbf{v}_{L}, \mathbf{v}_{R}\right)=\left\{\begin{array}{ccc}
\mathbf{u}_{1 L} & \text { if } & \frac{x}{t}<\lambda_{1}^{r}\left(\mathbf{v}_{L}\right), \\
\mathbf{u}_{1-} & \text { if } & \lambda_{1}^{r}\left(\mathbf{v}_{L}\right)<\frac{x}{t}<u_{2}, \\
\mathbf{u}_{1+} & \text { if } & u_{2}<\frac{x}{t}<\lambda_{2}^{r}\left(\mathbf{v}_{R}\right), \\
\mathbf{u}_{1 R} & \text { if } & \frac{x}{t}>\lambda_{2}^{r}\left(\mathbf{v}_{R}\right),
\end{array}\right. \\
\mathbf{u}_{2}\left(x / t, \mathbf{v}_{L}, \mathbf{v}_{R}\right)=\left\{\begin{array}{ccc}
\mathbf{u}_{2 L} & \text { if } & \frac{x}{t}<\lambda_{3}^{r}\left(\mathbf{v}_{L}\right), \\
\mathbf{u}_{2-} & \text { if } & \lambda_{3}^{r}\left(\mathbf{v}_{L}\right)<\frac{x}{t}<u_{2}, \\
\mathbf{u}_{2+} & \text { if } & u_{2}<\frac{x}{t}<\lambda_{4}^{r}\left(\mathbf{v}_{R}\right), \\
\mathbf{u}_{2 R} & \text { if } & \frac{x}{t}>\lambda_{4}^{r}\left(\mathbf{v}_{R}\right),
\end{array}\right.
\end{gathered}
$$

$$
X\left(x / t, \mathbf{v}_{L}, \mathbf{v}_{R}\right)=\left\{\begin{array}{ll}
X_{L} & \text { if } x<u_{2} t, \\
X_{R} & \text { if } x>u_{2} t,
\end{array} \quad \text { for } \quad X=\alpha_{1}, \mathcal{T}_{1}, \mathcal{T}_{2} .\right.
$$


In these formulas, $u_{2}$ denotes the speed of propagation of the contact associated with $\lambda_{0}^{r}$ and satisfies

$$
u_{2}=u_{2-}=u_{2+}
$$

Defining $u_{*}$ and $(\alpha u)_{*}$ by

$$
\left\{\begin{array}{l}
u_{*}=\frac{1}{2}\left(u_{1 L}+u_{1 R}\right)-\frac{1}{2 a_{1}}\left(\Pi_{1 R}-\Pi_{1 L}\right) \\
(\alpha u)_{*}=\frac{1}{2}\left(\alpha_{1 L} u_{1 L}+\alpha_{1 R} u_{1 R}\right)-\frac{1}{2 a_{1}}\left(\alpha_{1 R} \Pi_{1 R}-\alpha_{1 L} \Pi_{1 L}\right),
\end{array}\right.
$$

the four intermediate states

$$
\mathbf{u}_{1 \pm}=\left(\begin{array}{c}
\alpha_{1} \rho_{1} \\
\alpha_{1} \rho_{1} u_{1}
\end{array}\right)_{ \pm}, \quad \mathbf{u}_{2 \pm}=\left(\begin{array}{c}
\alpha_{2} \rho_{2} \\
\alpha_{2} \rho_{2} u_{2}
\end{array}\right)_{ \pm},
$$

are characterized by

and, setting

$$
\left\{\begin{array}{c}
u_{2}=\frac{\alpha_{2 L} \lambda_{3}^{r}\left(\mathbf{v}_{L}\right)+\alpha_{2 R} \lambda_{4}^{r}\left(\mathbf{v}_{R}\right)}{\alpha_{2 L}+\alpha_{2 R}}+\frac{\alpha_{2 L} I_{2 L}-\alpha_{2 R} I_{2 R}}{a_{2}\left(\alpha_{2 L}+\alpha_{2 R}\right)}+\frac{\overline{\left[\alpha_{2} \Pi_{2}\right]}\left(\mathbf{u}_{L}, \mathbf{u}_{R}\right)}{a_{2}\left(\alpha_{2 L}+\alpha_{2 R}\right)} \\
\tau_{2-}:=\frac{1}{\rho_{2-}}=\frac{u_{2}-\lambda_{3}^{r}\left(\mathbf{v}_{L}\right)}{a_{2}}, \quad \tau_{2+}:=\frac{1}{\rho_{2+}}=\frac{\lambda_{4}^{r}\left(\mathbf{v}_{R}\right)-u_{2}}{a_{2}} \\
I_{2 L}=p_{2}\left(1 / \mathcal{T}_{2 L}\right)+a_{2}^{2} \mathcal{T}_{2 L}, \quad I_{2 R}=p_{2}\left(1 / \mathcal{T}_{2 R}\right)+a_{2}^{2} \mathcal{T}_{2 R}
\end{array}\right.
$$

$$
\begin{gathered}
m:=\alpha_{1 L} \rho_{1-}\left(u_{1-}-u_{2}\right)=\alpha_{1 R} \rho_{1+}\left(u_{1+}-u_{2}\right) \\
=\frac{-\overline{\left[\alpha_{2} \Pi_{2}\right]}\left(\mathbf{u}_{L}, \mathbf{u}_{R}\right)+a_{1}\left(2(\alpha u)_{*}-u_{2}\left(\alpha_{1 L}+\alpha_{1 R}\right)\right)}{\lambda_{2}^{r}\left(\mathbf{v}_{R}\right)-\lambda_{1}^{r}\left(\mathbf{v}_{L}\right)} \\
\left\{\begin{array}{c}
u_{1-}=\frac{-a_{1} \overline{\left[\alpha_{2} \Pi_{2}\right]}\left(\mathbf{u}_{L}, \mathbf{u}_{R}\right)-\mathcal{M}_{\Pi}+2 a_{1}\left(a_{1}(\alpha u)_{*}-m u_{*}\right)}{2 a_{1}\left(a_{1} \alpha_{1 L}-m\right)}, \quad \tau_{1-}=\frac{u_{1-}-\lambda_{1}^{r}\left(\mathbf{v}_{L}\right)}{a_{1}} \\
u_{1+}=\frac{-a_{1} \overline{\left[\alpha_{2} \Pi_{2}\right]}\left(\mathbf{u}_{L}, \mathbf{u}_{R}\right)+\mathcal{M}_{\Pi}+2 a_{1}\left(a_{1}(\alpha u)_{*}+m u_{*}\right)}{2 a_{1}\left(a_{1} \alpha_{1 R}+m\right)}, \quad \tau_{1+}=\frac{\lambda_{2}^{r}\left(\mathbf{v}_{R}\right)-u_{1+}}{a_{1}} \\
\mathcal{M}_{\Pi}=m\left(I_{1 R}-I_{1 L}\right)+a_{1}^{2} u_{2}\left(\alpha_{1 R}-\alpha_{1 L}\right)
\end{array}\right. \\
I_{1 L}=p_{1}\left(1 / \mathcal{T}_{1 L}\right)+a_{1}^{2} \mathcal{T}_{1 L}, \quad I_{1 R}=p_{1}\left(1 / \mathcal{T}_{1 R}\right)+a_{1}^{2} \mathcal{T}_{1 R}
\end{gathered}
$$

The proof of this result is given in Appendix 1.

Remark 5.5. We underline that, by definition of the phase space $\Omega^{r}$, the volume fraction $\alpha_{1} \neq\{0,1\}$. Moreover, since the free parameters $a_{k}, k=1,2$ are chosen to guarantee that (5.13) hold, we get that $\rho_{1-}, \rho_{2-}, \rho_{1+}$ and $\rho_{2+}$ are necessarily positive.

\subsection{Stability and accuracy properties of the relaxation method}

The aim of this section is to give some interesting accuracy and stability properties of the proposed relaxation method. Their validity naturally relies on similar properties satisfied by the approximate Riemann solution $(x, t) \mapsto \tilde{\mathbf{v}}_{\mathbf{r}}\left(\frac{x}{t} ; \mathbf{v}_{L}, \mathbf{v}_{R}\right)$, and allow to validate our approach from a theoretical point of view. The next section will be devoted to numerical experiments.

We begin by collecting the main properties of the approximate Riemann solution. 
Proposition 5.6 (the Riemann solution $(x, t) \mapsto \tilde{\mathbf{v}}_{\mathbf{r}}\left(\frac{x}{t} ; \mathbf{v}_{L}, \mathbf{v}_{R}\right)$ ). Let $\mathbf{u}_{L}$ and $\mathbf{u}_{R}$ be two constant states in $\Omega$, and let $\mathbf{v}_{L}$ and $\mathbf{v}_{R}$ be the two corresponding equilibrium states defined by (5.8).

(i) (Conservativity). The Riemann solution $(x, t) \mapsto \tilde{\mathbf{v}}_{\mathbf{r}}\left(\frac{x}{t} ; \mathbf{v}_{L}, \mathbf{v}_{R}\right)$ satisfies the following three conservation laws if $\alpha_{1 L} \neq \alpha_{1 R}$ :

$$
\left\{\begin{array}{l}
\partial_{t} \alpha_{1} \rho_{1}+\partial_{x} \alpha_{1} \rho_{1} u_{1}=0 \\
\partial_{t} \alpha_{2} \rho_{2}+\partial_{x} \alpha_{2} \rho_{2} u_{2}=0 \\
\partial_{t}\left(\alpha_{1} \rho_{1} u_{1}+\alpha_{2} \rho_{2} u_{2}\right)+\partial_{x}\left(\alpha_{1} \rho_{1} u_{1}^{2}+\alpha_{2} \rho_{2} u_{2}^{2}+\alpha_{1} \Pi_{1}+\alpha_{2} \Pi_{2}\right)=0
\end{array}\right.
$$

and the following four conservation laws when $\alpha_{1 L}=\alpha_{1 R}$ :

$$
\left\{\begin{array}{l}
\partial_{t} \alpha_{1} \rho_{1}+\partial_{x} \alpha_{1} \rho_{1} u_{1}=0 \\
\partial_{t}\left(\alpha_{1} \rho_{1} u_{1}\right)+\partial_{x}\left(\alpha_{1} \rho_{1} u_{1}^{2}+\alpha_{1} \Pi_{1}\right)=0 \\
\partial_{t} \alpha_{2} \rho_{2}+\partial_{x} \alpha_{2} \rho_{2} u_{2}=0 \\
\partial_{t}\left(\alpha_{2} \rho_{2} u_{2}\right)+\partial_{x}\left(\alpha_{2} \rho_{2} u_{2}^{2}+\alpha_{2} \Pi_{2}\right)=0
\end{array}\right.
$$

In other words, the mass and momentum of both phases evolve according to natural conservation laws when expected.

(ii) (L $L^{1}$ stability). Provided that the free parameters $a_{1}=a_{1}\left(\mathbf{u}_{L}, \mathbf{u}_{R}\right)$ and $a_{2}=a_{2}\left(\mathbf{u}_{L}, \mathbf{u}_{R}\right)$ are chosen sufficiently large according to (5.13) and to (5.14), the Riemann solution $(x, t) \mapsto \tilde{\mathbf{v}}_{\mathbf{r}}\left(\frac{x}{t} ; \mathbf{v}_{L}, \mathbf{v}_{R}\right)$ belongs to $\Omega^{r}$.

(iii) (Solid contact discontinuity). Assume that $\mathbf{u}_{L}$ and $\mathbf{u}_{R}$ can be joined by an admissible contact discontinuity of the equilibrium system (1.2), i.e. are such that the jump relations (2.2) hold. Then, the approximate Riemann solution $(x, t) \mapsto \tilde{\mathbf{v}}_{\mathbf{r}}\left(\frac{x}{t} ; \mathbf{v}_{L}, \mathbf{v}_{R}\right)$ is exact in the sense that it consists of an isolated contact discontinuity separating $\mathbf{v}_{L}$ and $\mathbf{v}_{R}$ and propagating with speed $u_{2 L}=u_{2 R}$.

Proof.

(i) This is true by construction, see indeed systems (5.9).

(ii) We have to check that $\alpha_{k}\left(\frac{x}{t} ; \mathbf{v}_{L}, \mathbf{v}_{R}\right) \in(0,1)$ and $\rho_{k}\left(\frac{x}{t} ; \mathbf{v}_{L}, \mathbf{v}_{R}\right)>0$ with $k=1,2$ and for all $(x, t)$, $t>0$. The first point is obvious since $\alpha_{k}$ evolves according to a transport equation and then equals either $\alpha_{k L}$ or $\alpha_{k R}$. Concerning the second point, we know by Theorem 5.4 that the sign of the densities $\rho_{1-}, \rho_{1+}, \rho_{2-}$ and $\rho_{2+}$ are respectively the same as the sign of $u_{1-}-\lambda_{1}^{r}\left(\mathbf{v}_{L}\right), \lambda_{2}^{r}\left(\mathbf{v}_{R}\right)-u_{1+}, u_{2}-\lambda_{3}^{r}\left(\mathbf{v}_{L}\right)$ and $\lambda_{4}^{r}\left(\mathbf{v}_{R}\right)-u_{2}$. It is then clear by definition of the eigenvalues $\lambda_{l}^{r}, l=1, \ldots, 4$ that these quantities are necessarily positive for large values of $a_{1}$ and $a_{2}$ (note that $u_{1 \pm}$ and $u_{2}$ remain bounded in this regime).

(iii) To prove this accuracy property, it is sufficient by uniqueness of the Riemann solution $(x, t) \mapsto \tilde{\mathbf{v}}_{\mathbf{r}}\left(\frac{x}{t} ; \mathbf{v}_{L}\right.$, $\mathbf{v}_{R}$ ) to verify that the discontinuity separating $\mathbf{v}_{L}$ and $\mathbf{v}_{R}$ and propagating with speed $u_{2 L}=u_{2 R}$ is admissible in the sense of Definition 5.3. The fourth equation of (5.12) is trivially satisfied. The equilibrium property (5.8) implies $\mathcal{T}_{1 L}=\tau_{1 L}$ and $\mathcal{T}_{1 R}=\tau_{1 R}$, but also $\Pi_{1 L}=p_{1}\left(\rho_{1 L}\right)$ and $\Pi_{1 R}=p_{1}\left(\rho_{1 R}\right)$. Invoking the jump relations (2.2), the first three equations in (5.12) are then also immediate. The last equation follows from the fact that $\overline{\left[\alpha_{2} \Pi_{2}\right]}\left(\mathbf{u}_{L}, \mathbf{u}_{R}\right)=\alpha_{2 R} \Pi_{2 R}-\alpha_{2 L} \Pi_{2 L}$ since $\mathbf{u}_{L}$ and $\mathbf{u}_{R}$ are joined by an admissible contact.

Remark 5.7. It is important to notice at this stage that the accuracy property (iii) would not be satisfied if the original conservation law $\partial_{t} \alpha_{1} \rho_{1} \mathcal{T}_{1}+\partial_{x} \alpha_{1} \rho_{1} \mathcal{T}_{1} u_{1}=0$ had been retained in system (5.9). Indeed, the fourth equation in (5.12) would read in this case $m \mathcal{T}_{1 L}=m \mathcal{T}_{1 R}$ that is $m \tau_{1 L}=m \tau_{1 R}$ by the equilibrium property (5.8). This relation is of course false in general for $\mathbf{u}_{L}$ and $\mathbf{u}_{R}$ such that the jump relations (2.2) hold.

The results of Proposition 5.6 then easily apply to get the following theorem giving the main properties satisfied by the relaxation method described in Section 5.1. Recall that the latter is based on the classical averaging procedure (5.6) of the juxtaposition of Riemann solutions $(x, t) \mapsto \tilde{\mathbf{v}}_{\mathbf{r}}\left(\frac{x}{t} ; \mathbf{v}_{j}, \mathbf{v}_{j+1}\right)$ (Step 1), followed by an instantaneous relaxation that only modifies the relaxation quantities $\mathcal{T}_{1 j}$ and $\mathcal{T}_{2 j}$ (Step 2). 
Theorem 5.8 (the relaxation method). The relaxation method described in Section 5.1:

(i) (Conservativity): is always conservative on $\alpha_{k} \rho_{k}, k=1,2$ and $\alpha_{1} \rho_{1} u_{1}+\alpha_{2} \rho_{2} u_{2}$, but also on $\alpha_{k} \rho_{k} u_{k}$, $k=1,2$ if $\alpha_{1 j}$ is constant for all $j$.

(ii) ( $L^{1}$ stability): provides numerical solutions that remain in the phase space provided that the free parameters $a_{k}=a_{k}\left(\mathbf{u}_{j}, \mathbf{u}_{j+1}\right), k=1,2$ are chosen sufficiently large according to (5.13) and to (5.14), for all $j$.

(iii) (Solid contact discontinuity): captures exactly the stationary admissible contact discontinuities of the equilibrium system (1.2).

Remark 5.9. Due to the averaging procedure (5.6), it is usual that only the stationary admissible contact discontinuity may be exactly computed.

Remark 5.10. Property $(i)$ gives the variables that are updated with a conservative formula from time $t^{n}$ to time $t^{n+1-}$ in the relaxation method. More generally, and using Green's formula, we easily get from (5.6) and (5.11) the following non conservative update formula

$$
\begin{gathered}
\mathbf{v}_{j}^{n+1-}=\mathbf{v}_{j}^{n}-\nu\left(\mathbf{g}_{j+1 / 2}^{-}-\mathbf{g}_{j-1 / 2}^{+}\right)-\frac{1}{\Delta x} \int_{x_{j-1 / 2}}^{x_{j+1 / 2}} \int_{t^{n}}^{t^{n}+\Delta t} \tilde{\mathbf{d}}\left(\tilde{\mathbf{v}}_{\nu}(x, t)\right) \partial_{x} \tilde{\mathbf{v}}_{\nu}(x, t) \mathrm{d} x \mathrm{~d} t \\
\text { with } \mathbf{g}_{j+1 / 2}^{ \pm}=\mathbf{g}\left(\tilde{\mathbf{v}}_{\mathbf{r}}\left(0^{ \pm} ; \mathbf{v}_{j}^{n}, \mathbf{v}_{j+1}^{n}\right)\right) .
\end{gathered}
$$

We recover in particular that the variables associated with a zero component in the non conservative product are treated in conservation form.

\subsection{A natural cheaper relaxation method}

The explicit knowledge of the Riemann solution $(x, t) \mapsto \tilde{\mathbf{v}}_{\mathbf{r}}\left(x / t, \mathbf{v}_{L}, \mathbf{v}_{R}\right)$ given in Theorem 5.4 relies on the first computation of $\overline{\left[\alpha_{2} \Pi_{2}\right]}\left(\mathbf{u}_{L}, \mathbf{u}_{R}\right)$. In order to define the reconstructed states $\overline{\mathbf{u}}_{L}$ and $\overline{\mathbf{u}}_{R}$ in (5.10), this one necessitates twice the resolution of the nonlinear system (2.2) associated with a contact of the equilibrium system (1.2). A natural modification leading to a cheaper strategy consists in performing the reconstruction procedure when using the jump relations (4.1) associated with the relaxation system (3.1). Recall indeed that the former leads to explicit formulas (see Thm. 4.3).

The consequence of such a modification is the loss of the accuracy property (iii) in Proposition 5.6 and Theorem 5.8 above. Indeed, the new reconstructed states $\overline{\mathbf{u}}_{L}$ and $\overline{\mathbf{u}}_{R}$ are in this case (generally) different from $\mathbf{u}_{L}$ and $\mathbf{u}_{R}$ when these states can be joined by an admissible contact of system (1.2) (i.e. satisfy (2.2)). Then $\overline{\left[\alpha_{2} \Pi_{2}\right]}\left(\mathbf{u}_{L}, \mathbf{u}_{R}\right)$ is no longer exact.

Note however that systems (2.2) and (4.1) lead to the same solution in the asymptotic regime of relative Mach numbers $\frac{m^{2}}{\alpha_{1-}^{2} \rho_{1-}^{2}}$ and $\frac{m^{2}}{\alpha_{1-}^{2} \rho_{1-}^{2}}$ tending to zero. Indeed, we first get $\rho_{1-}=\rho_{1+}$ for both systems, then $\mathcal{T}_{1-}=\tau_{1-}=\mathcal{T}_{1+}=\tau_{1+}$ by the equilibrium property in (4.1), so that the first three equations eventually coincide in (2.2) and (4.1). This justifies the proposed modification in the context of subsonic flows of interest in this paper.

\section{NUMERICAL EXPERIMENTS}

This section is devoted to numerical simulations of the two fluid two pressure diphasic model. For each test we compare four different Riemann solvers that we label Rusanov, Relax-exact, Relax(1), Relax(2).

Rusanov denotes a non conservative version of the Rusanov scheme which is explained in detail in [20].

The other solvers are based on the relaxation approach we presented. The first one, labeled Relax-exact uses the exact Riemann solution $(x, t) \mapsto \mathbf{v}_{\mathbf{r}}\left(\frac{x}{t} ; \mathbf{v}_{L}, \mathbf{v}_{R}\right)$ in formula (5.6). We recall that this implies to solve a fourth-order polynomial which is achieved by an iterative algorithm (see [19]). 
TABLE 1. Pure stationary contact - initial conditions.

\begin{tabular}{cccc}
\hline \hline & Unity & $L$ & $R$ \\
\hline$\alpha_{1}$ & - & 0.8 & 0.2 \\
$p_{1}$ & $10^{5} \mathrm{~Pa}$ & 1.25 & $p_{1}\left(\rho_{1 R}\right)$ \\
$p_{2}$ & $10^{5} \mathrm{~Pa}$ & 1.25 & $p_{2}\left(\rho_{2 R}\right)$ \\
$u_{1}$ & $\mathrm{~m} \cdot \mathrm{s}^{-1}$ & 50 & $u_{1 R}$ \\
$u_{2}$ & $\mathrm{~m} \cdot \mathrm{s}^{-1}$ & 0 & $u_{2 R}$ \\
\hline \hline
\end{tabular}

The last two schemes use the approximate Riemann solution $(x, t) \mapsto \tilde{\mathbf{v}}_{\mathbf{r}}\left(\frac{x}{t} ; \mathbf{v}_{L}, \mathbf{v}_{R}\right)$ and correspond to two different estimations of the term $\Pi_{1} \partial_{x} \alpha_{1}$. The method labeled Relax(1) estimates this term via the jump conditions (2.2) of the initial diphasic model (1.2).

The other (cheaper) method labeled Relax(2) uses an estimation via the jump conditions of the relaxation model (3.1) with $\lambda=0$ and is explained in Section 5.4.

Two kinds of simulations are proposed. The first one referred to as 'pressures out of equilibrium' focuses on system (1.2) without any extra source term. On the contrary, the second one referred to as 'pressure equilibrium' takes into account an additional pressure relaxation source term between phases 1 and 2 in the first equation of system (1.2).

\subsection{Pressures out of equilibrium}

Here, by the sake of simplicity, we consider the following equations of state:

$$
p_{k}\left(\rho_{k}\right)=A_{k} \rho_{k}^{\gamma_{k}}, \quad k=1,2,
$$

which means that the two phases are isentropic. We note $\gamma_{k}$ the adiabatic coefficient of phase $k$ and $A_{k}$ a constant depending on the entropy of the fluid. Actually, $A_{k}$ is computed on the basis of a reference state: $A_{k}=\frac{p_{k 0}}{\rho_{k 0}^{\gamma k}}$. We consider a case where the coefficient $A_{k}$ takes the constant value of $10^{5} \mathrm{~Pa}\left(\frac{\mathrm{m}^{3}}{\mathrm{~kg}}\right)^{\gamma_{k}}$, for $k=1,2$ and we choose $\gamma_{1}=1.4$ and $\gamma_{2}=1.2$. Computations are performed on 200 cells. Initial conditions correspond to shock tube test cases:

$$
\mathbf{u}_{0}(x)= \begin{cases}\mathbf{u}_{L} & \text { if } x<10 \\ \mathbf{u}_{R} & \text { if } x>10\end{cases}
$$

\subsubsection{Pure stationary contact}

In this first test case, we consider a left state $\mathbf{u}_{L}$ and a right void fraction $\alpha_{1 R}$ given in Table 1 . Using the notations of Definition 2.2 we set the right state as $\mathbf{u}_{R}=\mathbf{u}_{+}\left(\mathbf{u}_{L}, \alpha_{1 R}\right)$. Numerical values of the remaining variables result from a resolution (for instance with a Newton algorithm) of system (2.2). Since the velocity of the left state is chosen to be zero, we have that the velocity of the right state is equal to zero. Thus, the two states $\mathbf{u}_{L}$ and $\mathbf{u}_{R}$ are separated by a stationary $\lambda_{0}$-contact discontinuity. This choice allows us to observe how each method preserves numerically such a solution. Results for the two phases are displayed at final time $0.02 \mathrm{~s}$ in Figures 3 and 4. In agreement with Theorem 5.8 above, the relaxation solver Relax(1) provides a perfectly sharp discontinuity at $x=10 \mathrm{~m}$ while the two other relaxation methods slightly diffuse the profile. The Rusanov scheme is even more diffusive. In particular, one can easily notice in Figure 4(b) that only Relax(1) exactly preserves the stationary profile of velocity $u_{2}$. The other solvers yield non uniform discrete solutions.

This test case illustrates numerically the importance of the term $m\left(\mathcal{T}_{1 R}-\mathcal{T}_{1 L}\right) \delta_{x-u_{2}^{*} t}$ added in (5.9) in the equation associated with $\alpha_{1} \rho_{1} \mathcal{T}_{1}$. Recall that this term is responsible for the approximate Riemann solution $(x, t) \mapsto \tilde{\mathbf{v}}_{\mathbf{r}}\left(\frac{x}{t} ; \mathbf{v}_{L}, \mathbf{v}_{R}\right)$ to be exact when an isolated contact discontinuity is considered. Indeed, the Relax(1) method provides an exact solution. In contrast, the Relax-exact method which does not take into account this modification leads to an approximate solution. 


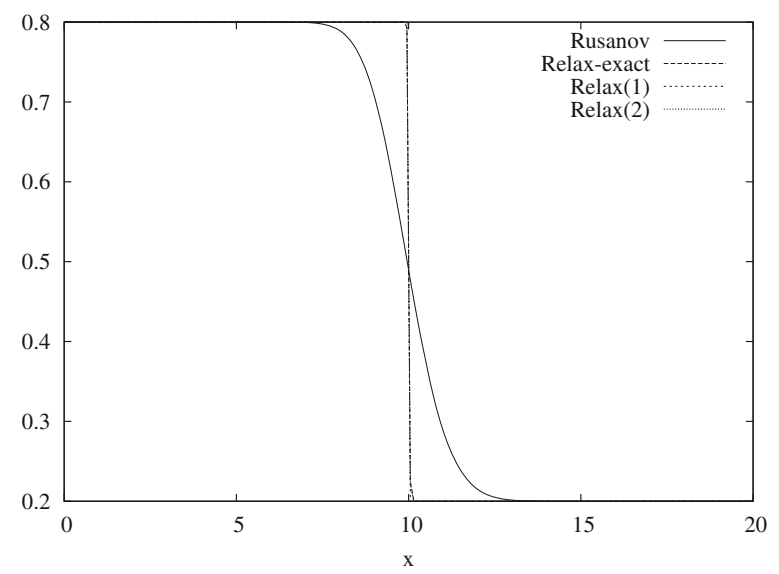

(a) Fraction $\alpha_{1}(-)$

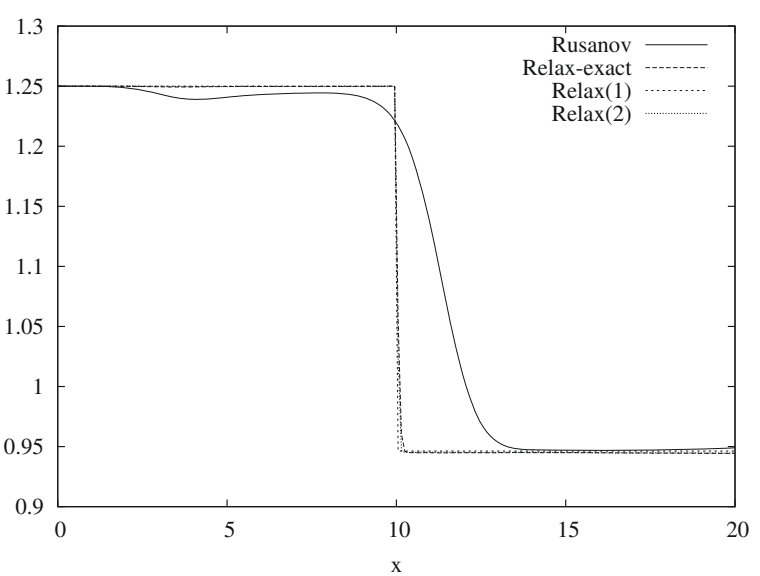

(b) Pressure $p_{1}\left(10^{5} \mathrm{~Pa}\right)$

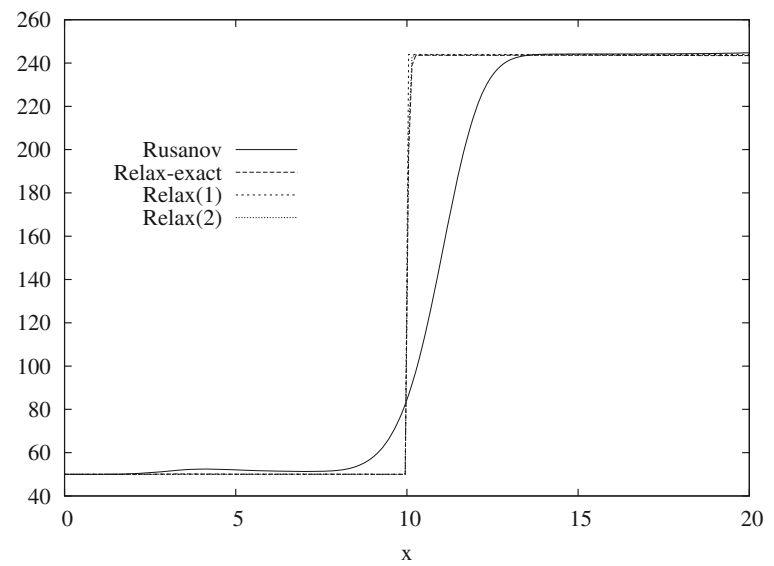

(c) Velocity $u_{1}(\mathrm{~m} / \mathrm{s})$

Figure 3. Pure stationary contact - numerical results for phase 1. 


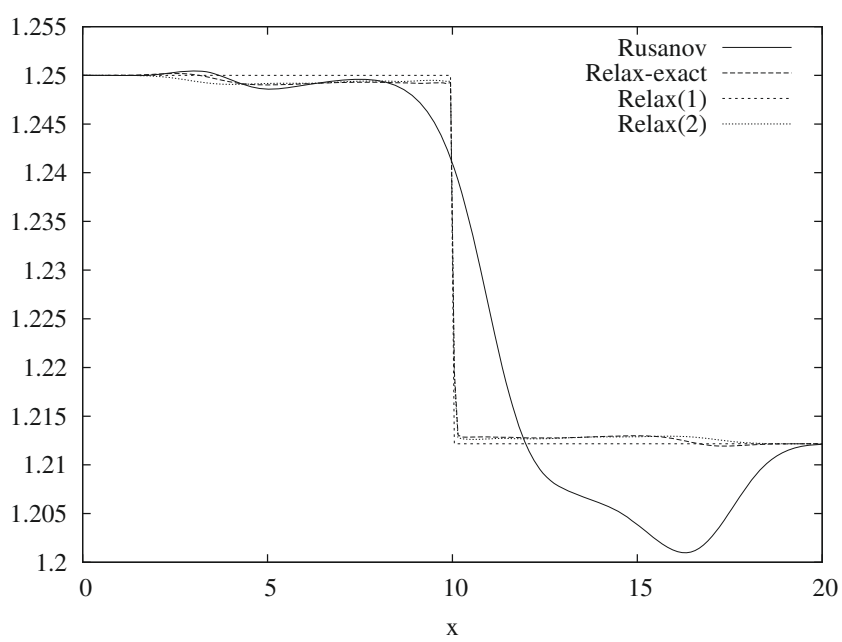

(a) Pressure $p_{2}\left(10^{5} \mathrm{~Pa}\right)$

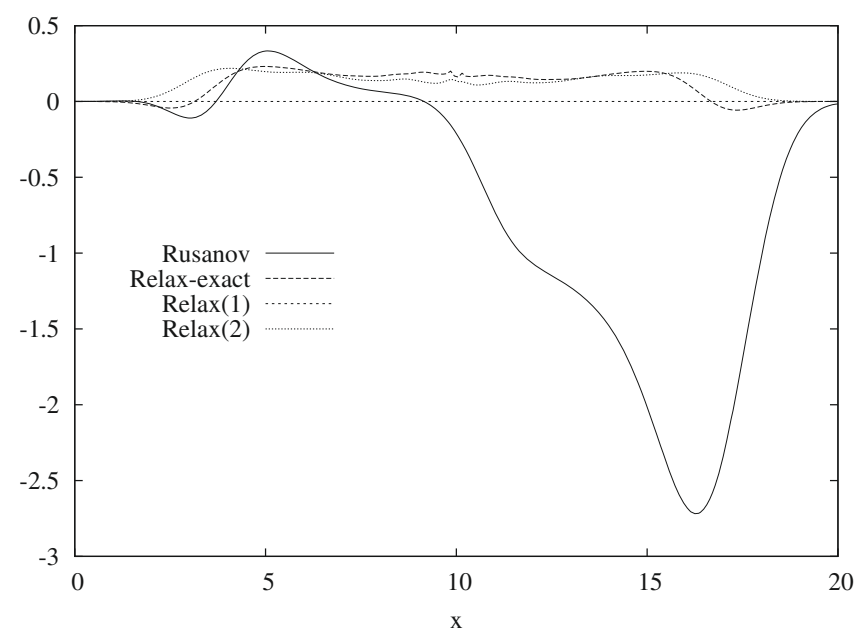

(b) Velocity $u_{2}(\mathrm{~m} / \mathrm{s})$

Figure 4. Pure stationary contact - numerical results for phase 2.

The failure of the Relax(2) solver has a different origin. This one actually takes into account the additional term $m\left(\mathcal{T}_{1 R}-\mathcal{T}_{1 L}\right) \delta_{x-u_{2}^{*} t}$ in $(5.9)$ but is based on an estimation of the non conservative term $\overline{\left[\alpha_{2} \Pi_{2}\right]}\left(\mathbf{u}_{L}, \mathbf{u}_{R}\right)$ via approximate jump conditions (4.1), instead of (2.2). This explains the non exact capture of the solution (see Sect. 5.4).

However, note that the errors are significantly smaller for the last two relaxation methods than for the Rusanov scheme.

\subsubsection{Moving contact discontinuity}

The initial conditions of this test case are given in Table 2. Data are constant except for the void fraction $\alpha_{1}$. Since we have $p_{1}=p_{2}=1.25 \times 10^{5} \mathrm{~Pa}$ and $u_{1}=u_{2}=50 \mathrm{~m} \cdot \mathrm{s}^{-1}$, the two states $\mathbf{u}_{L}$ and $\mathbf{u}_{R}$ verify the jump conditions $(2.2)$ with $m=0$. In other terms, the two states are separated by a pure moving $\lambda_{0}$-contact discontinuity of velocity $u_{2}=50 \mathrm{~m} \cdot \mathrm{s}^{-1}$. The results are plotted at time $0.05 \mathrm{~s}$ in Figures 5 and 6 . We can easily 


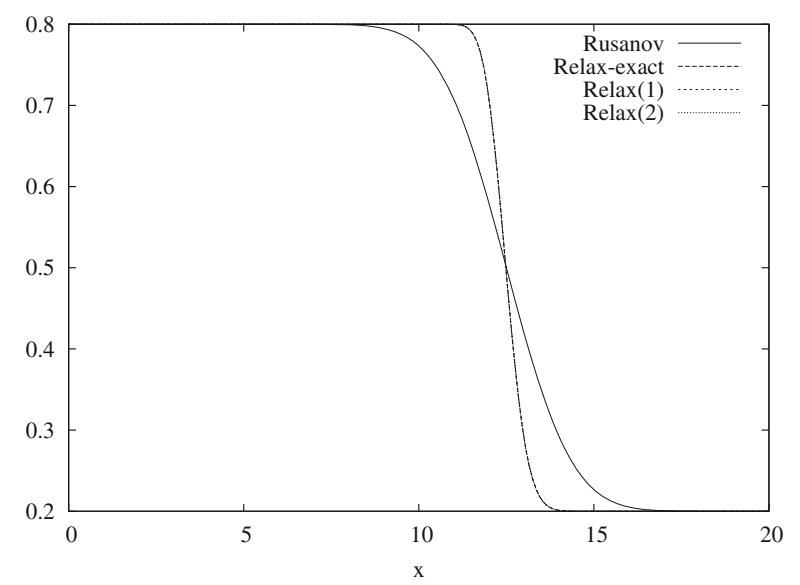

(a) Fraction $\alpha_{1}(-)$

Figure 5. Moving contact (1/2).

TABLE 2. Moving contact - initial conditions.

\begin{tabular}{cccc}
\hline \hline & Unity & $L$ & $R$ \\
\hline$\alpha_{1}$ & - & 0.8 & 0.2 \\
$p_{1}$ & $10^{5} \mathrm{~Pa}$ & 1.25 & 1.25 \\
$p_{2}$ & $10^{5} \mathrm{~Pa}$ & 1.25 & 1.25 \\
$u_{1}$ & $\mathrm{~m} \cdot \mathrm{s}^{-1}$ & 50 & 50 \\
$u_{2}$ & $\mathrm{~m} \cdot \mathrm{s}^{-1}$ & 50 & 50 \\
\hline \hline
\end{tabular}

TABLE 3. General shock tube test - initial conditions.

\begin{tabular}{cccc}
\hline \hline & Unity & $L$ & $R$ \\
\hline$\alpha_{1}$ & - & 0.8 & 0.2 \\
$p_{1}$ & $10^{5} \mathrm{~Pa}$ & 1.5 & 1.0 \\
$p_{2}$ & $10^{5} \mathrm{~Pa}$ & 1.5 & 1.0 \\
$u_{1}$ & $\mathrm{~m} \cdot \mathrm{s}^{-1}$ & 50 & 50 \\
$u_{2}$ & $\mathrm{~m} \cdot \mathrm{s}^{-1}$ & 5 & 5 \\
\hline \hline
\end{tabular}

notice that uniform profiles are preserved by all the methods. On the other hand, one checks that relaxationbased solvers yield similar results for the void fraction profile in Figure 5. They are obviously much less diffusive than the Rusanov scheme.

\subsubsection{General shock tube test}

Initial conditions of this shock tube test are given in Table 3. Results of this simulation are displayed at final time $0.01 \mathrm{~s}$ in Figures 7 and 8. All the solvers based on the relaxation approach give similar results. Again, one may remark that the relaxation approach is much less diffusive than the Rusanov method.

A second simulation is shown in Figure 9 and corresponds to a general shock tube test, where we compare results obtained using the two different estimates of $\overline{\left[\alpha_{2} \Pi_{2}\right]}$ presented above. For fine meshes (400 cells here), no difference can be spotted. 


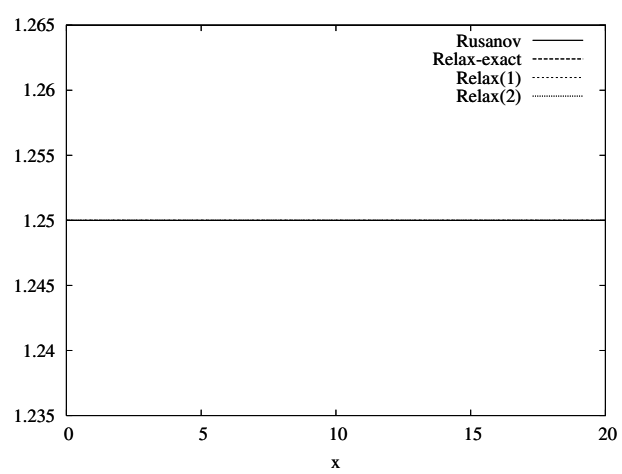

(a) Pressure $p_{1}\left(10^{5} \mathrm{~Pa}\right)$

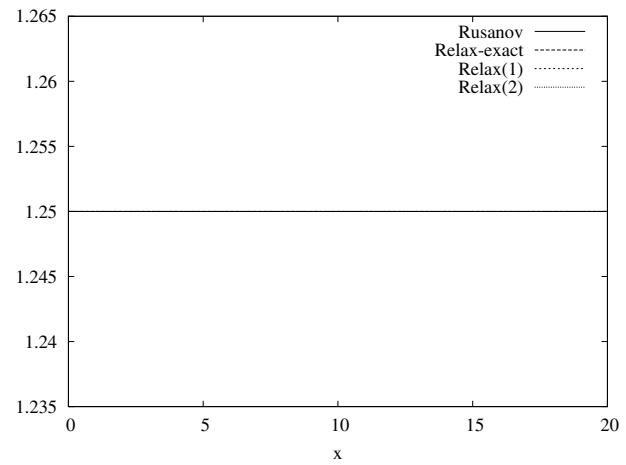

(c) Pressure $p_{2}\left(10^{5} \mathrm{~Pa}\right)$

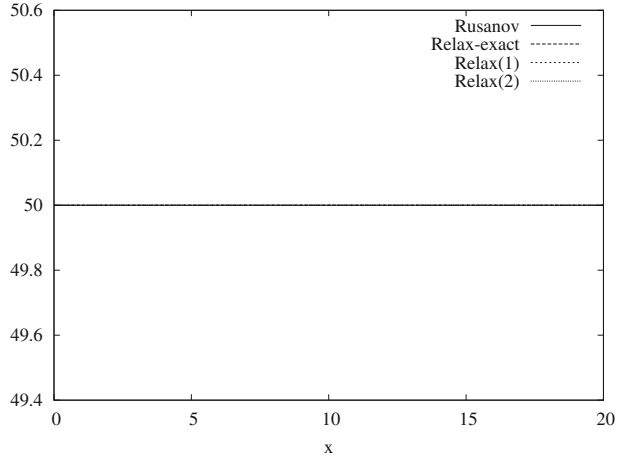

(b) Velocity $u_{1}(\mathrm{~m} / \mathrm{s})$

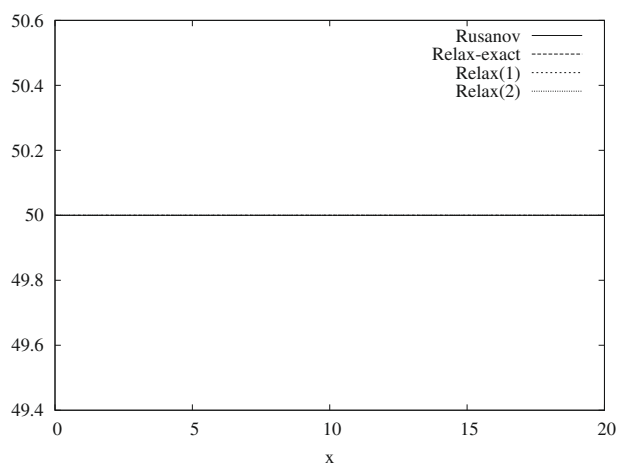

(d) Velocity $u_{2}(\mathrm{~m} / \mathrm{s})$

Figure 6. Moving contact discontinuity $(2 / 2)$.

TABLE 4. Constants in the equation of state.

\begin{tabular}{ccc}
\hline & $c_{k}\left(\mathrm{~m} \cdot \mathrm{s}^{-1}\right)$ & $\rho_{k}^{0}\left(\mathrm{~kg} \cdot \mathrm{m}^{-3}\right)$ \\
\hline Air (1) & $\sqrt{10^{5}}$ & 0 \\
Water (2) & 1000 & 999.9 \\
\hline
\end{tabular}

\subsection{Pressure equilibrium}

We want to solve in this section system (1.2) with the presence of an additional pressure relaxation source term in the first equation. More precisely, given $\varepsilon_{p}>0$ the pressure relaxation time between the two phases, this equation becomes

$$
\partial_{t} \alpha_{1}+u_{2} \partial_{x} \alpha_{1}=\frac{1}{\varepsilon_{p}}\left(p_{1}\left(\rho_{1}\right)-p_{2}\left(\rho_{2}\right)\right)
$$

We are interested in the asymptotic regime $\varepsilon_{p} \rightarrow 0$, so that equation (6.2) equivalently recasts, at least formally, $p_{1}\left(\rho_{1}\right)=p_{2}\left(\rho_{2}\right)$. Numerically, this instantaneous pressure relaxation is done via a two step splitting method detailed in the following.

First, consider the approximate solution $\mathbf{u}_{\nu}\left(x, t^{n}\right)$ at time $t^{n}$. We solve the convective part of the system on the whole space domain thanks to one of the proposed Riemann solvers and note the resulting approximate 


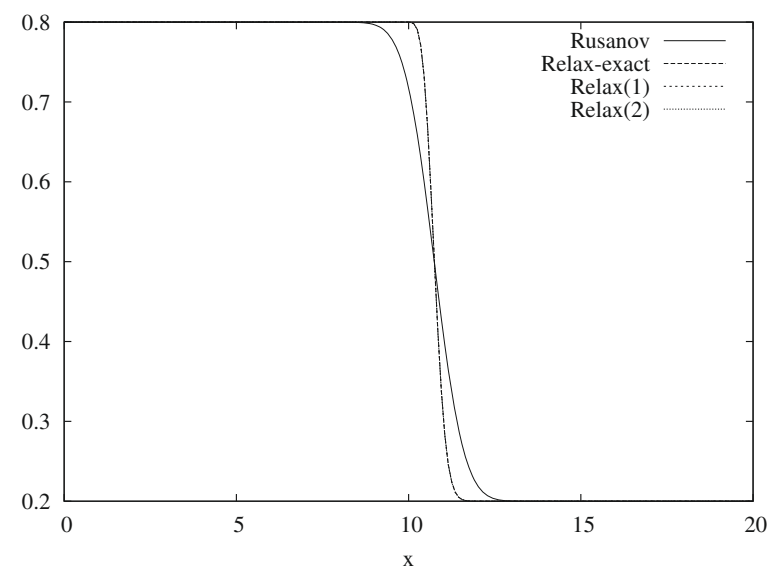

(a) Fraction $\alpha_{1}(-)$

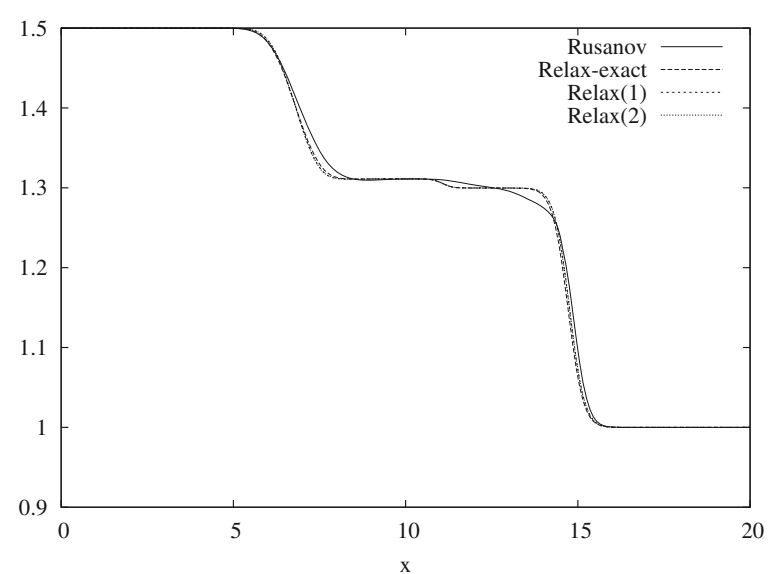

(b) Pressure $p_{1}\left(10^{5} \mathrm{~Pa}\right)$

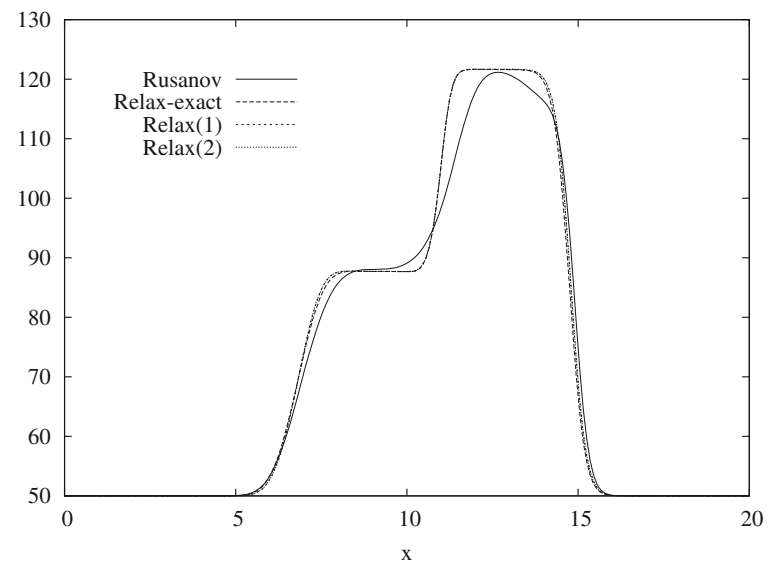

(c) Velocity $u_{1}(\mathrm{~m} / \mathrm{s})$

Figure 7. General shock tube test - numerical results for phase 1. 


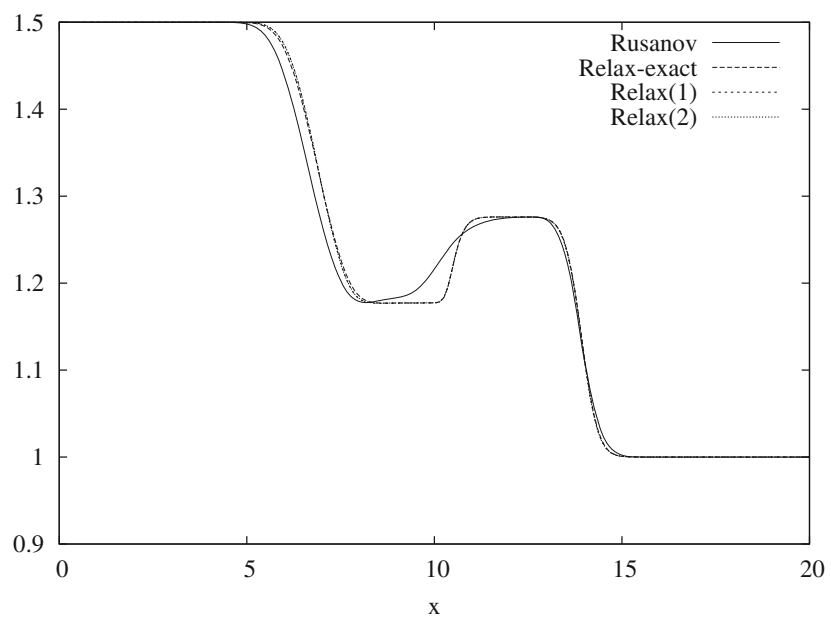

(a) Pressure $p_{2}\left(10^{5} \mathrm{~Pa}\right)$

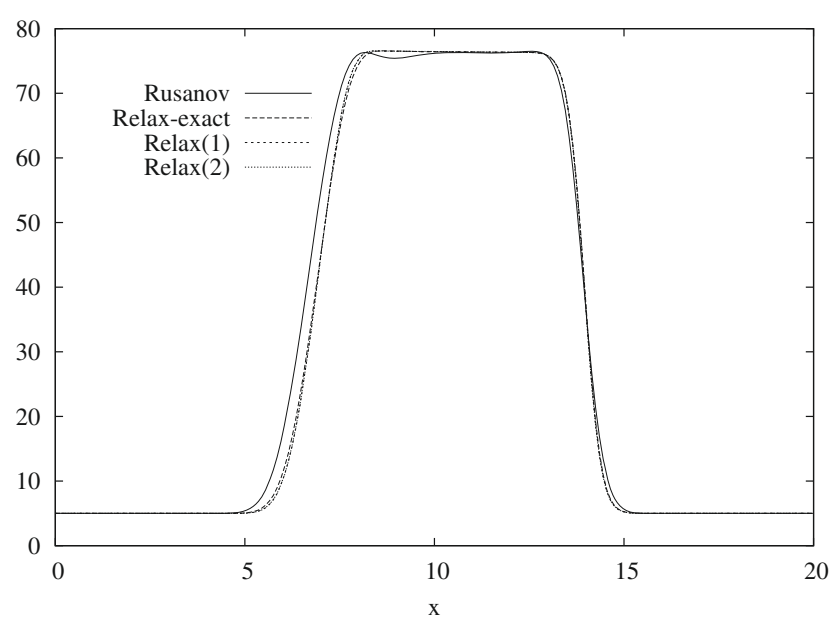

(b) Velocity $u_{2}(\mathrm{~m} / \mathrm{s})$

FiguRE 8. General shock tube test - numerical results for phase 2.

solution $\mathbf{u}_{\nu}\left(x, t^{n, 1}\right)$. Then the solution $\mathbf{u}_{\nu}\left(x, t^{n+1}\right)$ at time $t^{n+1}$ is obtained by setting

$$
\begin{aligned}
& \left(\alpha_{k} \rho_{k}\right)_{j}^{n+1}=\left(\alpha_{k} \rho_{k}\right)_{j}^{n, 1}, \\
& \left(\alpha_{k} \rho_{k} u_{k}\right)_{j}^{n+1}=\left(\alpha_{k} \rho_{k} u_{k}\right)_{j}^{n, 1}, \quad k=1,2,
\end{aligned}
$$

and resolving on each cell the following equation:

$$
p_{1}\left(\frac{\left(\alpha_{1} \rho_{1}\right)_{j}^{n+1}}{\left(\alpha_{1}\right)_{j}^{n+1}}\right)-p_{2}\left(\frac{\left(\alpha_{2} \rho_{2}\right)_{j}^{n+1}}{1-\left(\alpha_{1}\right)_{j}^{n+1}}\right)=0,
$$

to define the equilibrium void fraction $\left(\alpha_{1}\right)_{j}^{n+1}$. The equations of state we consider are the same than the ones given by Munkejord [32] that is

$$
p_{k}=c_{k}^{2}\left(\rho_{k}-\rho_{k}^{0}\right), \quad k=1,2
$$

where the constants $c_{k}$ and $\rho_{k}^{0}$ are respectively the sound speed and the 'reference density' of phase $k$. The values of these constants are given in Table 4. Equation (6.3) can be solved explicitly. We get the equilibrium void fraction $\left(\alpha_{1}\right)_{j}^{n+1}$ at time $t^{n+1}$ by the following formula:

$$
\left(\alpha_{2}\right)_{j}^{n+1}=1-\left(\alpha_{1}\right)_{j}^{n+1}=\frac{-\psi_{2}-\sqrt{\psi_{2}^{2}-4 \psi_{1} \psi_{3}}}{2 \psi_{1}},
$$

where

$$
\begin{aligned}
& \psi_{1}=c_{2}^{2} \rho_{2}^{0}-c_{1}^{2} \rho_{1}^{0}, \\
& \psi_{2}=-c_{2}^{2}\left(\left(\alpha_{2} \rho_{2}\right)_{j}^{n+1}+\rho_{2}^{0}\right)+c_{1}^{2}\left(-\left(\alpha_{1} \rho_{1}\right)_{j}^{n+1}+\rho_{1}^{0}\right),
\end{aligned}
$$

and

$$
\psi_{3}=c_{2}^{2}\left(\alpha_{2} \rho_{2}\right)_{j}^{n+1}
$$




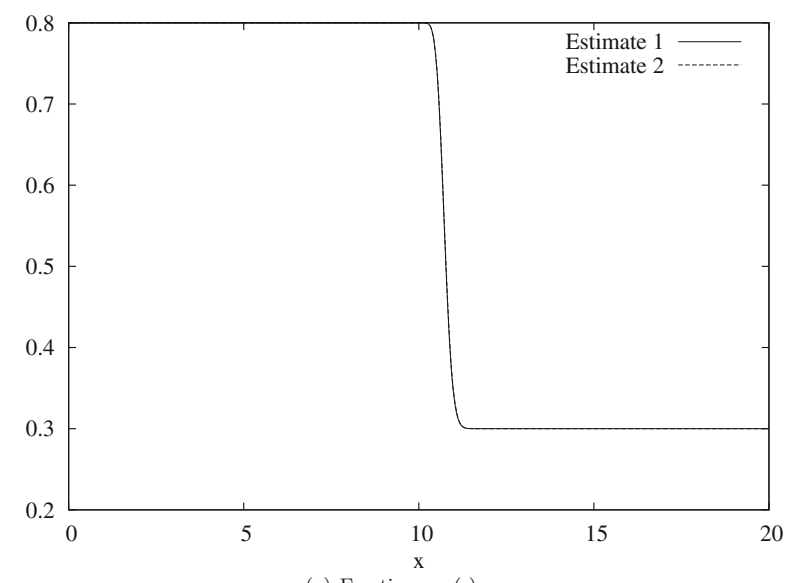

(a) Fraction $\alpha_{1}(-)$

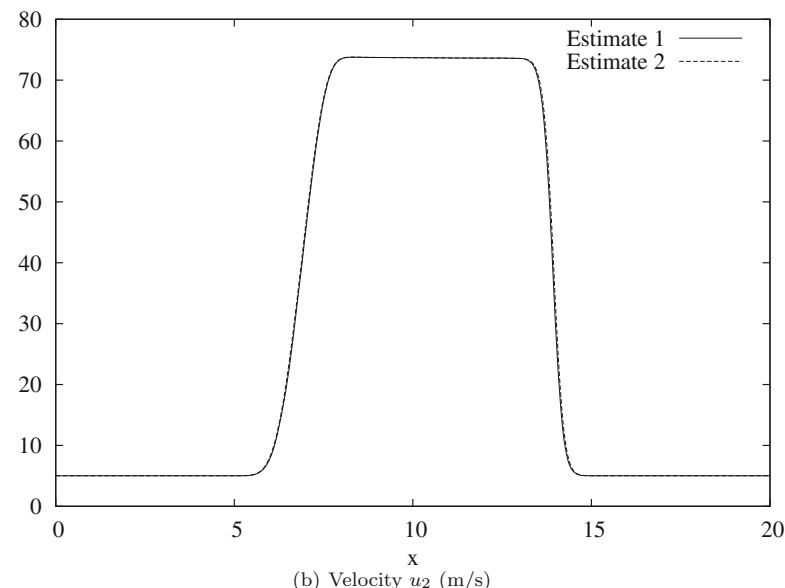

Figure 9. Shock tube-different estimate of $\overline{\left[\alpha_{2} \Pi_{2}\right]}$.

TABLE 5. Large relative velocity shock tube - initial conditions.

\begin{tabular}{cccc}
\hline \hline & Unity & $L$ & $R$ \\
\hline$\alpha_{1}$ & - & 0.29 & 0.30 \\
$p$ & $10^{5} \mathrm{~Pa}$ & 2.65 & 2.65 \\
$u_{1}$ & $\mathrm{~m} \cdot \mathrm{s}^{-1}$ & 65 & 50 \\
$u_{2}$ & $\mathrm{~m} \cdot \mathrm{s}^{-1}$ & 1 & 1 \\
\hline \hline
\end{tabular}

\subsubsection{Large relative velocity shock tube}

This test case is given in Munkejord [32]. The length of the domain is now $100 \mathrm{~m}$ and initial conditions are such that:

$$
\mathbf{u}_{0}(x)= \begin{cases}\mathbf{u}_{L} & \text { if } x<50 \\ \mathbf{u}_{R} & \text { if } x>50\end{cases}
$$




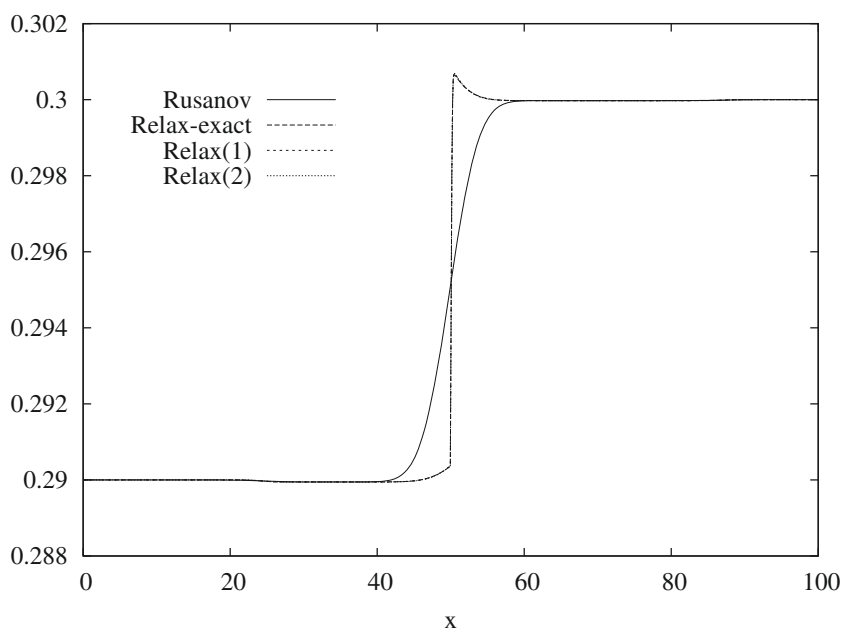

(a) Fraction $\alpha_{1}(-)$

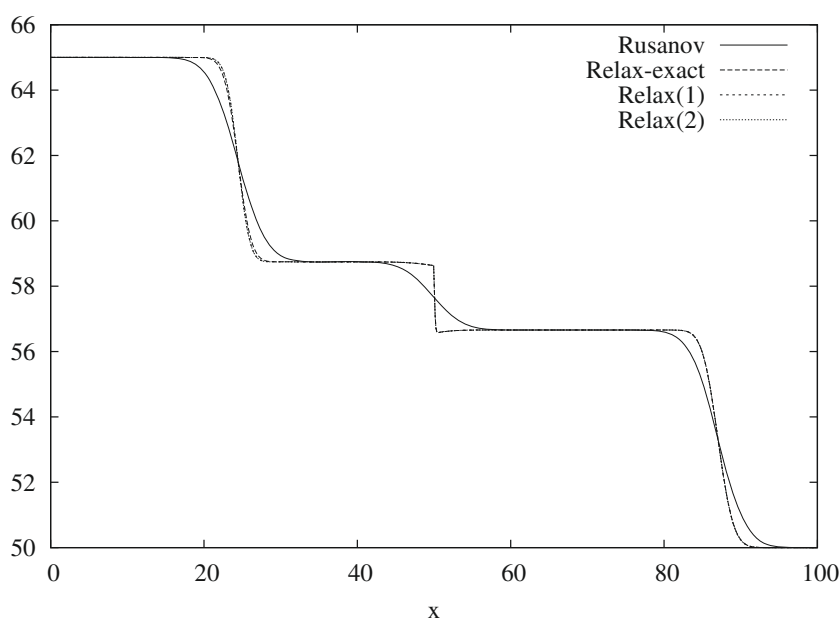

(b) Velocity $u_{1}(\mathrm{~m} / \mathrm{s})$

FiguRE 10. Large relative velocity shock tube - results for phase 1.

TABLE 6 . The water faucet test case - inlet and outlet conditions.

\begin{tabular}{cccc}
\hline \hline & Unity & Inlet & Outlet \\
\hline$\alpha_{1}$ & - & 0.2 & - \\
$p$ & $10^{5} \mathrm{~Pa}$ & 1.0 & 1.0 \\
$u_{1}$ & $\mathrm{~m} \cdot \mathrm{s}^{-1}$ & 0 & - \\
$u_{2}$ & $\mathrm{~m} \cdot \mathrm{s}^{-1}$ & 10 & - \\
\hline \hline
\end{tabular}

with $\mathbf{u}_{L}$ and $\mathbf{u}_{R}$ written in Table 5. We compute the solution on a grid of 1000 cells. Results are plotted at final time $0.1 \mathrm{~s}$ in Figures 10 and 11. These numerical results are similar to the one obtained in [32] with a first-order scheme. 


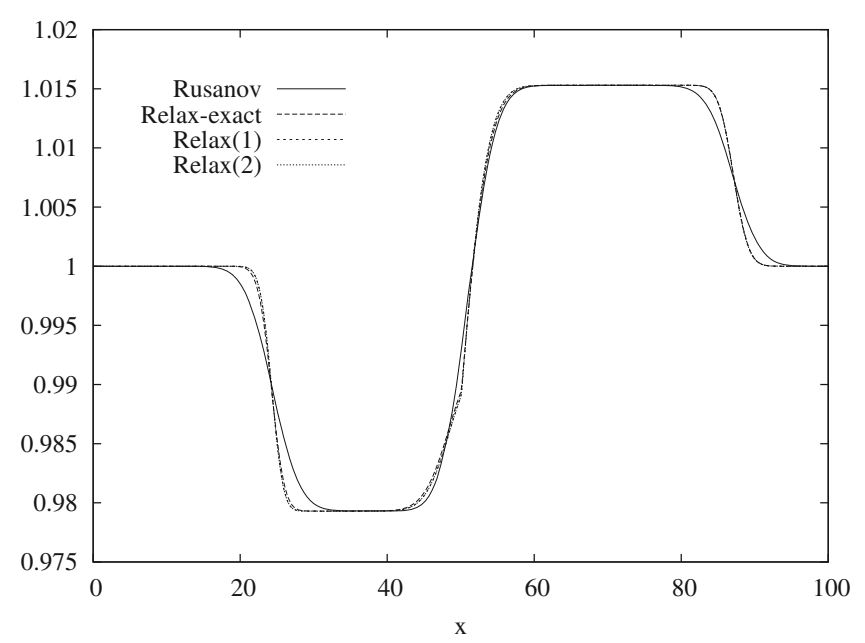

(a) Velocity $u_{2}(\mathrm{~m} / \mathrm{s})$

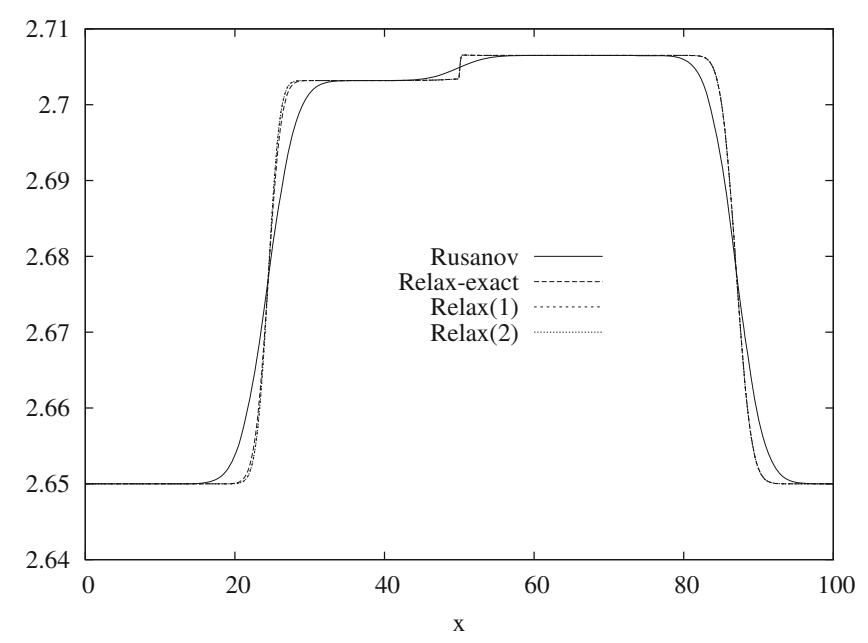

(b) Pressure $p\left(10^{5} \mathrm{~Pa}\right)$

FiguRE 11. Large relative velocity shock tube - results for phase 2.

\subsubsection{The water faucet test case}

The water faucet test case is a classical benchmark test for one-dimensional two-phase flow simulations and is described in Ransom [33]. This test consists in an incoming water flow in presence of gravity in a vertical tube with length $12 \mathrm{~m}$ and diameter $1 \mathrm{~m}$. More precisely, at the temperature $T=50{ }^{\circ} \mathrm{C}$, we consider a flow of water (phase 2) at the top of the tube in $x=0 \mathrm{~m}$ with inlet conditions given in Table 6 . The bottom of the tube in $x=12 \mathrm{~m}$ is open to room pressure $p=10^{5} \mathrm{~Pa}$ (outlet conditions) and the top of the tube is closed to air flow (phase 1), i.e.

$$
u_{1}(x=0, t)=0 .
$$

The initial conditions on the domain are given by the inlet conditions. Since a gravity field is introduced in this test, the momentum balance equation for phase $k$ reads

$$
\partial_{t}\left(\alpha_{k} \rho_{k} u_{k}\right)+\partial_{x}\left(\alpha_{k} \rho_{k} u_{k}^{2}+p_{k}\left(\rho_{k}\right)\right)-p_{1}\left(\rho_{1}\right) \partial_{x} \alpha_{k}=\alpha_{k} \rho_{k} g, \quad k=1,2 .
$$


In the numerical scheme, the source term $\alpha_{k} \rho_{k} g$ is accounted for by using a standard explicit centered approximation. We fix the boundary conditions to the inlet conditions for the top of the tube while we solve an half Riemann problem for the bottom (see Dubois and LeFloch [17]). Due to the effect of gravity, a thin liquid jet will form. It is common to compare the solution of this problem to the analytical solution of the much simpler model corresponding to the following momentum balance

$$
\partial_{t}\left(\alpha_{2} \rho_{2} u_{2}\right)+\partial_{x}\left(\alpha_{2} \rho_{2} u_{2}^{2}\right)=\alpha_{2} \rho_{2} g
$$

This gives for the air void fraction (see [14]):

$$
\alpha_{1}(x, t)= \begin{cases}1-\frac{\alpha_{2}^{0} u_{2}^{0}}{\sqrt{2 g x+\left(u_{2}^{0}\right)^{2}},}, & \text { if } x \leq u_{2}^{0} t+\frac{1}{2} g t^{2}, \\ 1-\alpha_{2}^{0}, & \text { otherwise }\end{cases}
$$

where $\alpha_{2}^{0}=\alpha_{2}(x, t=0)$ and $u_{2}^{0}=u_{2}(x, t=0)$ and for the velocity of water:

$$
u_{2}(x, t)= \begin{cases}\sqrt{\left(u_{2}^{0}\right)^{2}+2 g x}, & \text { if } x \leq u_{2}^{0} t+\frac{1}{2} g t^{2} \\ u_{2}^{0}+g t, & \text { otherwise }\end{cases}
$$

Numerical results for Relax(2) method are plotted in Figures 12(a) and 12(b) with different grid resolutions. Since the other relaxation-based solvers give similar results, we plot results only for the scheme Relax(2). The results are similar to Munkejord [32] for a first order scheme on the two-fluid two-pressure diphasic model with instantaneous pressure relaxation.

\section{Appendix 1. Proof of Theorem 5.4}

First of all we recall that the eigenvalues and the nature of the characteristic fields of system (5.9) are the same than the ones of system (3.5).

Moreover, from the first equation in (5.9), we deduce that the variable $\alpha_{1}$ (and then $\alpha_{2}$, since $\alpha_{2}=1-\alpha_{1}$ ) admits a jump only on the $\lambda_{0}$-contact discontinuity. Then, on the left and on the right of this discontinuity, we can rewrite system (5.9) as two decoupled systems on the fluid 1 and fluid 2 respectively:

$$
\begin{aligned}
& \left\{\begin{array}{l}
\partial_{t} \rho_{1}+\partial_{x}\left(\rho_{1} u_{1}\right)=0, \\
\partial_{t}\left(\rho_{1} u_{1}\right)+\partial_{x}\left(\rho_{1} u_{1}^{2}+\Pi_{1}\right)=0, \quad \text { for } \quad \frac{x}{t}<\lambda_{0}^{r} \quad \text { and } \quad \frac{x}{t}>\lambda_{0}^{r}, \\
\partial_{t}\left(\rho_{1} \mathcal{T}_{1}\right)+\partial_{x}\left(\rho_{1} \mathcal{T}_{1} u_{1}\right)=0,
\end{array}\right. \\
& \left\{\begin{array}{l}
\partial_{t} \rho_{2}+\partial_{x}\left(\rho_{2} u_{2}\right)=0, \\
\partial_{t}\left(\rho_{2} u_{2}\right)+\partial_{x}\left(\rho_{2} u_{2}^{2}+\Pi_{2}\right)=0, \\
\partial_{t}\left(\rho_{2} \mathcal{T}_{2}\right)+\partial_{x}\left(\rho_{2} \mathcal{T}_{2} u_{2}\right)=0,
\end{array} \quad \text { for } \quad \frac{x}{t}<\lambda_{0}^{r} \quad \text { and } \quad \frac{x}{t}>\lambda_{0}^{r} .\right.
\end{aligned}
$$

At the $\lambda_{0}$-contact discontinuity, the jump relations (5.12) must be verified.

We focus now on the variable $\mathcal{T}_{1}$. It appears from (6.7) and (5.12), that jumps in $\mathcal{T}_{1}$ are admissible only on the $\lambda_{0}$ and the $\lambda_{5}$ wave. The fourth equation in (5.12) implies that the whole jump between the right and left values is carried by this latter wave. Therefore, $\mathcal{T}_{1}$ is continuous on the $\lambda_{5}$-wave and the same is true for all the other variables by usual jump conditions on (6.7) and (6.8). This implies that the Riemann problem solution does not depend on the relative position of the $\lambda_{5^{-}}$and $\lambda_{0}$-waves. Its structure is presented in Figure 13 . We want now to compute the intermediate states for the fluid 2. The Rankine-Hugoniot relations on the momentum balance equation on the $\lambda_{3}$ and $\lambda_{4}$-waves read:

$$
\begin{aligned}
-\lambda_{3}^{r}\left(\mathbf{v}_{L}\right)\left(\rho_{2-} u_{2-}-\rho_{2 L} u_{2 L}\right)+\left(\rho_{2-} u_{2-}^{2}-\rho_{2 L} u_{2 L}^{2}\right)+\Pi_{2-}-\Pi_{2 L}=0, \\
-\lambda_{4}^{r}\left(\mathbf{v}_{R}\right)\left(\rho_{2 R} u_{2 R}-\rho_{2+} u_{2+}\right)+\left(\rho_{2 R} u_{2 R}^{2}-\rho_{2+} u_{2+}^{2}\right)+\Pi_{2 R}-\Pi_{2+}=0 .
\end{aligned}
$$




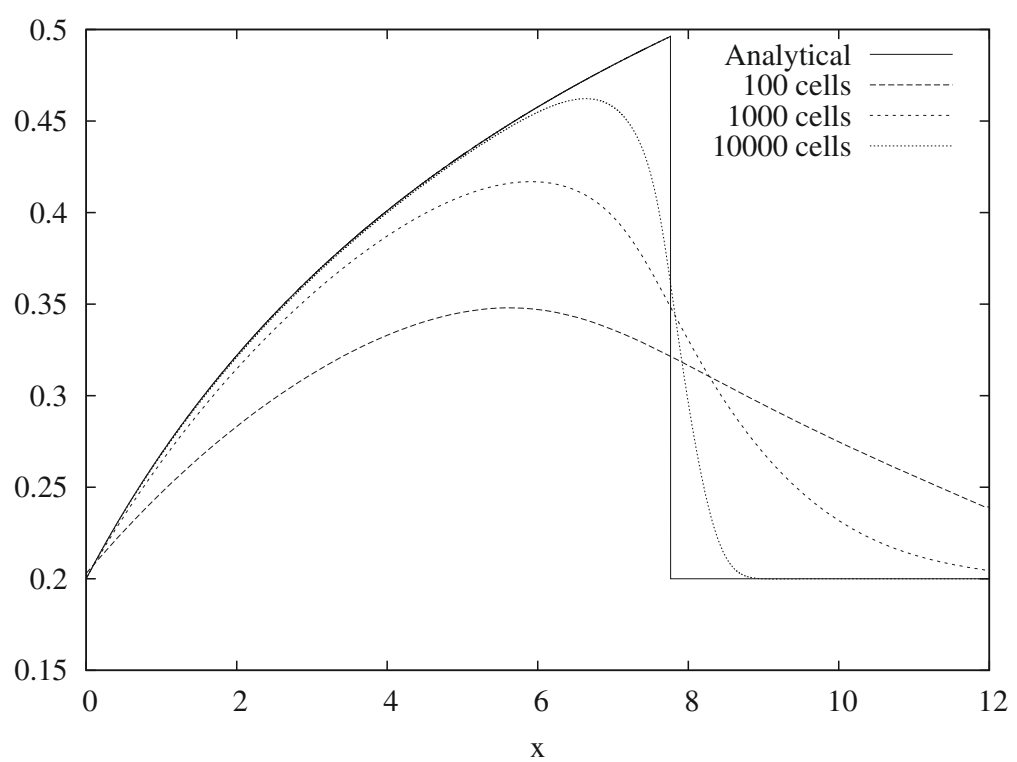

(a) Fraction $\alpha_{1}(-)$

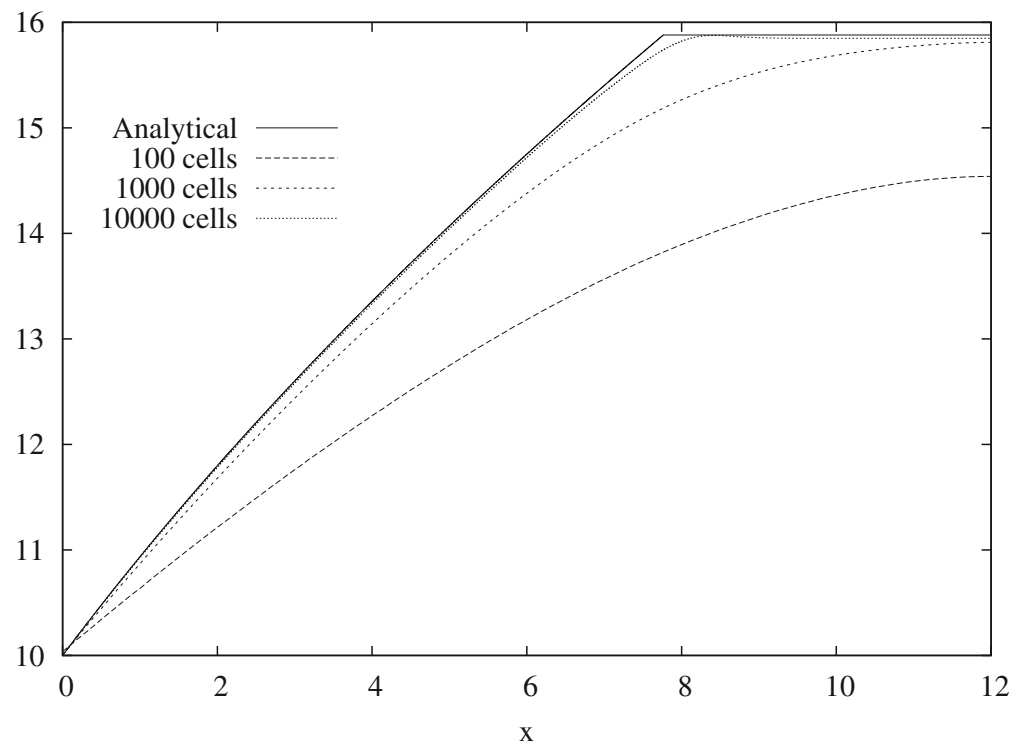

(b) Velocity $u_{2}(\mathrm{~m} / \mathrm{s})$

Figure 12. The water faucet test case - numerical results for Relax(2).

Whereas the continuity of the wave speeds for the $\lambda_{3}, \lambda_{4}$ and $\lambda_{0}$-waves gives:

$$
\begin{aligned}
& \lambda_{0}^{r}=u_{2-}=u_{2+}=u_{2}, \\
& \lambda_{3}^{r}\left(\mathbf{v}_{L}\right)=u_{2 L}-a_{2} \tau_{2 L}=u_{2}-a_{2} \tau_{2-}, \\
& \lambda_{4}^{r}\left(\mathbf{v}_{R}\right)=u_{2 R}+a_{2} \tau_{2 R}=u_{2}+a_{2} \tau_{2+} .
\end{aligned}
$$




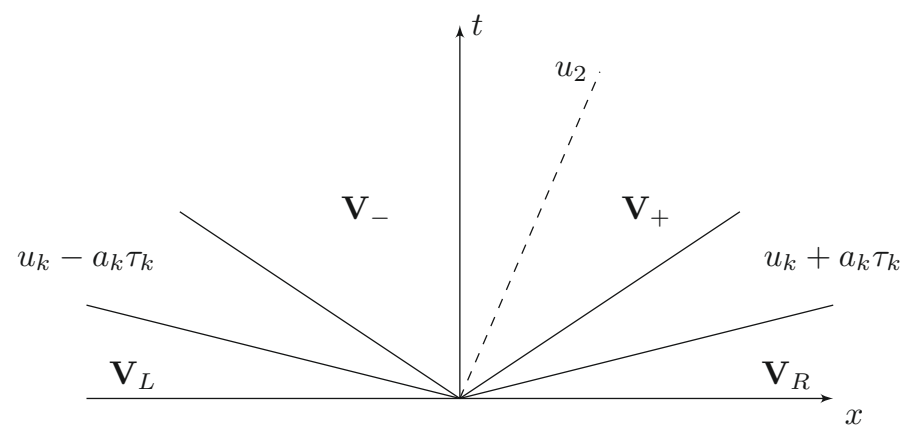

FIGURE 13. General structure in the subsonic regime of the Riemann solution associated with (5.9).

Using these relations, the Rankine-Hugoniot jump conditions above become:

$$
\begin{aligned}
a_{2}\left(u_{2}-u_{2 L}\right) & =\Pi_{2 L}-\Pi_{2-}, \\
a_{2}\left(u_{2}-u_{2 R}\right) & =\Pi_{2+}-\Pi_{2 R} .
\end{aligned}
$$

We multiply the first of these equations by $\alpha_{2 L}$, the second by $\alpha_{2 R}$ and sum them. By using the last relation in (5.12), we finally obtain

$$
u_{2}=\frac{\alpha_{2 L} \lambda_{3}^{r}\left(\mathbf{v}_{L}\right)+\alpha_{2 R} \lambda_{4}^{r}\left(\mathbf{v}_{R}\right)}{\alpha_{2 L}+\alpha_{2 R}}+\frac{\alpha_{2 L} I_{2 L}-\alpha_{2 R} I_{2 R}}{a_{2}\left(\alpha_{2 L}+\alpha_{2 R}\right)}+\frac{\overline{\left[\alpha_{2} \Pi_{2}\right]}\left(\mathbf{u}_{L}, \mathbf{u}_{R}\right)}{a_{2}\left(\alpha_{2 L}+\alpha_{2 R}\right)} .
$$

From (6.9), we have

$$
\tau_{2-}:=\frac{1}{\rho_{2-}}=\frac{u_{2}-\lambda_{3}^{r}\left(\mathbf{v}_{L}\right)}{a_{2}}, \quad \tau_{2+}:=\frac{1}{\rho_{2+}}=\frac{\lambda_{4}^{r}\left(\mathbf{v}_{R}\right)-u_{2}}{a_{2}} .
$$

We pass now to the computation of the intermediate states for the fluid 1 . The first step is to get the value of $m$. The continuity of the wave speeds for the $\lambda_{1}$ and $\lambda_{2}$-waves gives:

$$
\begin{aligned}
& \lambda_{1}^{r}\left(\mathbf{v}_{L}\right)=u_{1 L}-a_{1} \tau_{1 L}=u_{1-}-a_{1} \tau_{1-}, \\
& \lambda_{2}^{r}\left(\mathbf{v}_{R}\right)=u_{1 R}+a_{1} \tau_{1 R}=u_{1+}+a_{1} \tau_{1+},
\end{aligned}
$$

whereas by definition of $m$ one has:

$$
\left\{\begin{aligned}
\alpha_{1 L}\left(u_{1-}-u_{2}\right) & =m \tau_{1-} \\
\alpha_{1 R}\left(u_{1+}-u_{2}\right) & =m \tau_{1+}
\end{aligned}\right.
$$

Once $m$ is known, the quantities $\tau_{1-}, \tau_{1+}, u_{1-}, u_{1+}$ are then easily obtained by solving a linear system given by the four equations (6.11)-(6.12) and whose matrix is

$$
\left(\begin{array}{cccc}
-a_{1} & 0 & 1 & 0 \\
0 & a_{1} & 0 & 1 \\
m & 0 & -\alpha_{1 L} & 0 \\
0 & m & 0 & -\alpha_{1 R}
\end{array}\right)
$$

Its determinant is given by $\left(m-a_{1} \alpha_{1 L}\right)\left(m+a_{1} \alpha_{1 R}\right)$ and is negative provided that $a_{1}$ is chosen sufficiently large (see Rem. 5.5). Now the third and the fifth relations in (5.12) give

$$
m\left(u_{1+}-u_{1-}\right)+\left(\alpha_{1 R} \Pi_{1+}-\alpha_{1 L} \Pi_{1-}\right)=-\overline{\left[\alpha_{2} \Pi_{2}\right]}\left(\mathbf{u}_{L}, \mathbf{u}_{R}\right)
$$


Then we write

$$
\begin{aligned}
m\left(\lambda_{2}^{r}\left(\mathbf{v}_{R}\right)-\lambda_{1}^{r}\left(\mathbf{v}_{L}\right)\right) & =m\left(u_{1+}-u_{1-}\right)+a_{1} m\left(\tau_{1+}-\tau_{1-}\right) \\
& =-\overline{\left[\alpha_{2} \Pi_{2}\right]}\left(\mathbf{u}_{L}, \mathbf{u}_{R}\right)-\left(\alpha_{1 R} \Pi_{1+}-\alpha_{1 L} \Pi_{1-}\right)+a_{1} \alpha_{1 L}\left(u_{1-}-u_{2}\right)+a_{1} \alpha_{1 R}\left(u_{1+}-u_{2}\right) \\
& =-\overline{\left[\alpha_{2} \Pi_{2}\right]}\left(\mathbf{u}_{L}, \mathbf{u}_{R}\right)-a_{1} u_{2}\left(\alpha_{1 L}+\alpha_{1 R}\right)+\alpha_{1 L}\left(a_{1} u_{1-}+\Pi_{1-}\right)+\alpha_{1 R}\left(a_{1} u_{1+}-\Pi_{1+}\right) .
\end{aligned}
$$

But for the phase 1, the analogous of (6.10) writes $a_{1} u_{1-}+\Pi_{1-}=a_{1} u_{1 L}+\Pi_{1 L}$ and $a_{1} u_{1+}-\Pi_{1+}=a_{1} u_{1 R}-\Pi_{1 R}$. In other words, the right-hand side is known and as an immediate consequence $m$ also does. Easy calculations give the expected formulas for $u_{1-}, u_{1+}, \rho_{1-}, \rho_{1+}$ and $m$.

Acknowledgements. The work presented here was partially supported by the NEPTUNE project, with financial support from CEA (Commissariat à l'Énergie Atomique), EDF (Électricité de France), IRSN (Institut de Radioprotection et de Sûreté Nucléaire) and AREVA-NP.

\section{REFERENCES}

[1] R. Abgrall and R. Saurel, Discrete equations for physical and numerical compressible multiphase mixtures. J. Comput. Phys. 186 (2003) 361-396.

[2] A. Ambroso, C. Chalons, F. Coquel, T. Galié, E. Godlewski, P.-A Raviart and N. Seguin, The drift-flux asymptotic limit of barotropic two-phase two-pressure models. Comm. Math. Sci. 6 (2008) 521-529.

[3] N. Andrianov, Analytical and numerical investigation of two-phase flows. Ph.D. Thesis, Univ. Magdeburg, Germany (2003).

[4] N. Andrianov and G. Warnecke, The Riemann problem for the Baer-Nunziato two-phase flow model. J. Comput. Phys. 195 (2004) 434-464.

[5] N. Andrianov, R. Saurel and G. Warnecke, A simple method for compressible multiphase mixtures and interfaces. Int. J. Numer. Methods Fluids 41 (2003) 109-131.

[6] M.R. Baer and J.W. Nunziato, A two phase mixture theory for the deflagration to detonation (DDT) transition in reactive granular materials. Int. J. Multiphase Flows 12 (1986) 861-889.

[7] C. Berthon, B. Braconnier, B. Nkonga, Numerical approximation of a degenerate non-conservative multifluid model: relaxation scheme. Int. J. Numer. Methods Fluids 48 (2005) 85-90.

[8] F. Bouchut, Nonlinear stability of finite volume methods for hyperbolic conservation laws, and well-balanced schemes for sources, Frontiers in Mathematics series. Birkhauser (2004).

[9] B. Braconnier, Modélisation numérique d'écoulements multiphasiques pour des fluides compressibles, non miscibles et soumis aux effets capillaires. Ph.D. Thesis, Université Bordeaux I, France (2007).

[10] T. Buffard, T. Gallouët and J.M. Hérard, A sequel to a rough Godunov scheme. Application to real gas flows. Comput. Fluids 29 (2000) 813-847.

[11] C.E. Castro and E.F. Toro, A Riemann solver and upwind methods for a two-phase flow model in nonconservative form. Int. J. Numer. Methods Fluids 50 (2006) 275-307.

[12] C. Chalons and F. Coquel, Navier-Stokes equations with several independent pressure laws and explicit predictor-corrector schemes. Numer. Math. 101 (2005) 451-478.

[13] C. Chalons and J.F. Coulombel, Relaxation approximation of the Euler equations. J. Math. Anal. Appl. 348 (2008) $872-893$.

[14] F. Coquel, K. El Amine, E. Godlewski, B. Perthame and P. Rascle, Numerical methods using upwind schemes for the resolution of two-phase flows. J. Comput. Phys. 136 (1997) 272-288.

[15] F. Coquel, E. Godlewski, A. In, B. Perthame and P. Rascle, Some new Godunov and relaxation methods for two phase flows, in Proceedings of the International Conference on Godunov methods: theory and applications, Kluwer Academic, Plenum Publisher (2001).

[16] F. Coquel, T. Gallouët, J.M. Hérard and N. Seguin, Closure laws for a two-phase two-pressure model. C. R. Math. 334 (2002) 927-932.

[17] F. Dubois and P.G. LeFloch, Boundary conditions for nonlinear hyperbolic systems of conservation laws. J. Differ. Equ. 71 (1988) 93-122.

[18] P. Embid and M. Baer, Mathematical analysis of a two-phase continuum mixture theory. Contin. Mech. Thermodyn. 4 (1992) 279-312.

[19] T. Galié, Couplage interfacial de modèles en dynamique des fluides. Application aux écoulements diphasiques. Ph.D. Thesis, Université Pierre et Marie Curie, France (2008).

[20] T. Gallouët, J.M. Hérard and N. Seguin, Numerical modeling of two-phase flows using the two-fluid two-pressure approach. Math. Mod. Meth. Appl. Sci. 14 (2004) 663-700. 
[21] S. Gavrilyuk and R. Saurel, Mathematical and numerical modeling of two-phase compressible flows with micro-inertia. J. Comput. Phys. 175 (2002) 326-360.

[22] J. Glimm, D. Saltz and D.H. Sharp, Two phase flow modelling of a fluid mixing layer. J. Fluid Mech. 378 (1999) $119-143$.

[23] P. Goatin and P.G. LeFloch, The Riemann problem for a class of resonant nonlinear systems of balance laws. Ann. Inst. H. Poincaré, Anal. Non linéaire 21 (2004) 881-902.

[24] E. Godlewski and P.A. Raviart, Numerical approximation of hyperbolic systems of conservation laws. Springer-Verlag (1996).

[25] V. Guillemaud, Modélisation et simulation numérique des écoulements diphasiques par une approche bifluide à deux pressions. Ph.D. Thesis, Université de Provence, Aix-Marseille 1, France (2007).

[26] S. Jin and Z. Xin, The relaxation schemes for systems of conservation laws in arbitrary space dimensions. Comm. Pure Appl. Math. 48 (1995) 235-276.

[27] A.K. Kapila, S.F. Son, J.B. Bdzil, R. Menikoff and D.S. Stewart, Two phase modeling of DDT: structure of the velocityrelaxation zone. Phys. Fluids 9 (1997) 3885-3897.

[28] S. Karni, E. Kirr, A. Kurganov and G. Petrova, Compressible two-phase flows by central and upwind schemes. ESAIM: M2AN 38 (2004) 477-493.

[29] P.G. LeFloch, Entropy weak solutions to nonlinear hyperbolic systems in nonconservative form. Commun. Partial Differ. Equ. 13 (1988) 669-727.

[30] P.G. LeFloch, Shock waves for nonlinear hyperbolic systems in nonconservative form. Preprint \# 593, Institute for Math. and its Appl., Minneapolis, USA (1989).

[31] P.G. LeFloch and M.D. Thanh, The Riemann problem for fluid flows in a nozzle with discontinuous cross-section. Comm. Math. Sci. 1 (2003) 763-796.

[32] S.T. Munkejord, Comparison of Roe-type methods for solving the two-fluid model with and without pressure relaxation. Comput. Fluids 36 (2007) 1061-1080.

[33] V.H. Ransom, Numerical benchmark tests, in Multiphase science and technology, Vol. 3, G.F. Hewitt, J.M. Delhaye and N. Zuber Eds., Washington, USA, Hemisphere/Springer (1987) 465-467.

[34] V.V Rusanov, Calculation of interaction of non-steady shock waves with obstacles. J. Comp. Math. Phys. USSR 1 (1961) $267-279$.

[35] R. Saurel and R. Abgrall, A multiphase Godunov method for compressible multifluid and multiphase flows. J. Comput. Phys. 150 (1999) 425-467.

[36] R. Saurel and O. Lemetayer, A multiphase model for compressible flows with interfaces, shocks, detonation waves and cavitation. J. Fluid Mech. 431 (2001) 239-271.

[37] D.W. Schwendeman, C.W. Wahle and A.K Kapila, The Riemann problem and high-resolution Godunov method for a model of compressible two-phase flow. J. Comput. Phys. 212 (2006) 490-526. 